\title{
System for deployment of groups of unmanned micro aerial vehicles in GPS-denied environments using onboard visual relative localization
}

\author{
Martin Saska ${ }^{1, *}$, Tomas Baca ${ }^{1}$, Justin Thomas ${ }^{2}$, Jan Chudoba ${ }^{1}$, Libor Preucil ${ }^{1}$, Tomas Krajnik ${ }^{3}$, Jan Faigl ${ }^{1}$, \\ Giuseppe Loianno ${ }^{2}$, Vijay Kumar ${ }^{2}$
}

\begin{abstract}
A complex system for control of swarms of Micro Aerial Vehicles (MAV), in literature also called as Unmanned Aerial Vehicles (UAV) or Unmanned Aerial Systems (UAS), stabilized via an onboard visual relative localization is described in this paper. The main purpose of this work is to verify the possibility of self-stabilization of multi-MAV groups without an external global positioning system. This approach enables the deployment of MAV swarms outside laboratory conditions, and it may be considered an enabling technique for utilizing fleets of MAVs in real-world scenarios. The proposed visualbased stabilization approach has been designed for numerous different multi-UAV robotic applications (leader-follower UAV formation stabilization, UAV swarm stabilization and deployment in surveillance scenarios, cooperative UAV sensory measurement) in this paper. Deployment of the system in real-world scenarios truthfully verifies its operational constraints, given by limited onboard sensing suites and processing capabilities. The performance of the presented approach (MAV control, motion planning, MAV stabilization, and trajectory planning) in multiMAV applications has been validated by experimental results in indoor as well as in challenging outdoor environments (e.g., in windy conditions and in a former pit mine).
\end{abstract}

\section{INTRODUCTION}

The proposed approach relies strictly on onboard sensors and aspires to be an enabling technique for using closely cooperating MAV-groups in workspaces that are not equipped with motion capture systems (e.g. VICON ${ }^{1}$ ), which usually provide very precise and fast global localization of MAVs. With the proposed method, the utilization of closely cooperating MAVs is possible without installing any global localization infrastructure prior to the MAVs deployment in a GPS-denied environment. Besides, it enables applicability of multi-MAV teams in tasks requiring flight operations in close proximity between neighbors, where precision and reliability of GPS are not sufficient. The proposed approach is also especially appealing for missions in which the GPS signal may be jammed.

The robot localization being restricted to the onboard sensory system also significantly reduces the amount of communication necessary for the robots' coordination. In some applications, the group stabilization and control towards mission objectives can be achieved without explicit

\footnotetext{
${ }^{1}$ The authors are with the Dept. of Cybernetics, Faculty of Electrical Engineering, Czech Technical University in Prague, Technicka 2, 16627 Prague, Czech Republic.

${ }^{2}$ The authors are with the GRASP Lab, University of Pennsylvania, 3330 Walnut Street, 19104, Philadelphia, USA.

${ }^{3}$ The author is with the Lincoln Centre for Autonomous Systems, University of Lincoln, Brayford Pool, Lincoln, UK.

*martin.saskaefel.cvut.cz

1 http://www.vicon.com/
}

communication, as shown later in this paper where examples of the applicability of the system are presented. Disabled communication is crucial for MAVs operating in workspaces where radio transmissions are not feasible due to the structure of the environment or due to safety rules. Besides, current communication technologies do not provide sufficient bandwidth for large communities of robots operating in relatively small areas. In the proposed method, robots can share the information required for self-stabilization through observation of states of neighbors, i.e. by the onboard (in our case, visual) relative localization.

This paper presents a control system designed for multiMAV teams, its overall structure, and a description of its components. An important part of the paper is an overview of three commonly used planning approaches for multiMAV system (formation control, environment monitoring by swarm control, and MAV-group deployment in a surveillance scenario), which were designed for using with this system. In the description of the methods, it is highlighted how to deal with constraints given by the visual relative localization and how to integrate them into motion planning in specific multi-MAV applications. This should provide a guideline for developing high level planning algorithms in specific multi-MAV applications, since satisfying constraints of the onboard relative localization is crucial for achieving reliable behaviour by the MAV-group. Unlike the external global positioning system, where the precision and reliability of the robots' localization is independent to mutual positions of MAVs and the shape of the swarm, the operational space of the onboard relative localization sensors (for the vision sensor, mainly the range and the view angle of cameras) significantly limits the deployment of robots.

We rely on a light-weight embedded vision system using monocular cameras with a limited view angle. The system takes advantage of the possibility to equip all team members with black and white (B/W) patterns, which enables us to achieve sufficient precision on the order of centimeters if the actual distance between neighboring vehicles is on the order of meters. The detection of simple patterns with known shape and size also significantly speeds up the image processing. The localization system may therefore provide relative position measurements up to 60 times per second, and may be directly employed in the feedback loop for control and stabilization of the MAV-group.

The proposed control scheme integrates information from an onboard camera module with data from an inertial mea- 
surement unit and a commercially available PX4Flow ${ }^{2}$ smart sensor employed to measure the altitude and velocities of particular MAVs in the swarm. The MAV-group is then stabilized in three levels. The lowest level is the fastest control loop realized by the OEM MikroKopter's attitude stabilization board ${ }^{3}$ Above this loop, we have developed a position stabilization mechanism that leverages data from the visual relative localization unit in the control feedback. On the top of that, we show three examples of swarm motion planning. The motion planning acts as the third control level designed for navigation of the whole MAVgroup and its stabilization in required shapes, which may be dynamically changed. The methods employ a concept of adaptively evolving group behaviors that are established to decrease the uncertainty of the relative localization. These approaches are novel in the way how the constraints of vision based localization are incorporated into the control scheme. The operational constraints of the relative localization describe where neighboring particles or an object of interest equipped with the identification pattern may be detected and localized with a required precision and reliability. Plans that consider a model of the localization precision and reliability may decrease the overall uncertainty and increase the reliability of the complex autonomous system, as it was shown in our previous work on this topic [1]. Therefore, the proposed group motion planning approaches use a model of the localization system arising from theoretical analyses of the vision system and from an experimental evaluation of the system performance in real scenarios.

The paper is organized as follows. The related work and the contribution of the proposed MAV-group stabilization systems with respect to the state-of-the-art are presented in Section III In Section III] the hardware components of the localization module and the pattern detector approach are presented. The control scheme suited for onboard visual relative localization is proposed in Section IV. Section V presents an experimental verification of the system. Section VI summarizes three examples of high level motion control with integrated MAV motion constraints, obstacle avoidance, and constraints of the relative localization. These approaches and the performance of the onboard relative localization system are verified in real flight conditions. Finally, concluding remarks are stated in Section VII

\section{STATE-OF-THE-ART}

\section{A. Swarms of autonomous vehicles}

Recent research on multi-MAV systems has focused on aspects of communication and maintenance of connectivity within the team members [2], [3], modeling of the swarm behavior by predicting individual behaviors [4], [5], task allocation and strategies for solving multiple tasks [6], [7], [8], and control and collision avoidance within the swarm [9], [10], [11], [12]. Topics covered in this paper are related

\footnotetext{
http://pixhawk.org/modules/px4flow
} 3 http://www.mikrokopter.de/ mainly to control and stabilization of MAV teams. In literature, one can find papers describing control methodologies for swarms of both autonomous ground vehicles [13], [14], [15], [16] and unmanned aerial vehicles [17], [18], [19], [20]. These methods are often inspired by nature (e.g., by flocks of birds [21] or molecules forming crystals [22]), and they try to fulfil various requirements of swarm robotics. Since the proposed approach follows the requirements of swarms as listed in [23]: scalability for large groups, high redundancy and fault tolerance, usability in tasks unsolvable by a single robot and locally limited sensing and communication abilities, examples of studies investigating these domains should also be mentioned. In particular, a hierarchical framework for planning and control of arbitrarily large swarms is proposed in [13]. Considerations influencing the fault tolerance of teams are discussed in [24] and various co-operation strategies for teams of MAVs solving multi-robot tasks are published in [25]. Finally, controllers for swarms of robots with limited communication requirements are described in [14] and [15], where the necessary conditions for swarm stability are described using a direct graph topology in [14], and a Lyapunov-like function is employed for convergence analysis of multi-robot systems in [15].

The work in [15], which investigates swarming behaviors of ground robots in a planar environment, is the most closely related to the research proposed in this paper. We also aim to develop a system for stabilization of swarms in a desired shape while maintaining a close distance among swarm members. Beyond the method designed in [15] for ground robots, 3D swarm principles and swarming rules adapted for the requirements of visual relative localization are established in this paper.

In general, most of the state-of-the-art algorithms mentioned above have been verified only via numerical simulations, using ground vehicles, or rarely with MAVs, but in laboratory conditions (usually with VICON in control feedback). These approaches therefore often omit realistic constraints given by the real outdoor deployment of compact MAV-groups, which is the aim of this paper. The proposed system goes beyond these works mainly by incorporating the requirements of relative visual positioning into the MAVgroup motion planning, stabilization, and coordination. This improvement makes it possible to deploy large multi-MAV systems flying in compact formations or swarms outside of laboratories equipped with positioning systems. Besides, the possibility of direct interactions by perceiving neighboring robots in the MAV-group brings artificial swarms closer to the initial ideas and theoretical studies of swarming principles observed in nature.

\section{B. Systems of relative localization of autonomous robots}

Let us now briefly describe the state-of-the-art methods of geometric pattern detection, since the employed visual relative localization system based on $\mathrm{B} / \mathrm{W}$ pattern detection is instrumental in the presented control approach. A basic method for geometric pattern detection is the Generalized Hough Transform [26] used for finding the parameters of the 
expected geometrical shapes, which is unfortunately computationally demanding. The computational complexity issue is investigated e.g. in [27], where the RANSAC algorithm is applied, in [28], which is aimed at tracking objects easily separable from the background, and in [29], where the method is constrained to finding ellipses. These methods are sufficiently fast when using a standard PC, which may be placed onboard more powerful ground robots. However, these methods cannot be considered real-time for lightweight MAVs equipped with small embedded processors. One can find algorithms suited for embedded systems with real-time performance, but their limitations restrict their utilization in real-world applications (e.g. the system in [30], which is based on detecting color segments, and the approach in [31], which uses a pattern of four tennis balls, suffers in varying lighting conditions).

If we omit methods with image processing performed on an external desktop PC (e.g. [32], [33]), the most relevant approach to our vision system is proposed in [34]. The method [34] uses white rings for MAV positioning during landing, but provides a relative position update at only $0.1 \mathrm{~Hz}$. In addition, a more powerful onboard PC is required for the real-time control in [34]. The same problem arises in [35], where the " $\mathrm{H}$ " shape landing pattern is detected in realtime, but with a powerful onboard PC. Our solution provides sufficient sensitivity of detection and precision for the MAVgroup stabilization and satisfies computational requirements of onboard embedded systems carried by lightweight MAVs.

\section{SyStEM FOR RELATIVE LOCALIZATION}

As mentioned in the introduction, the core technique for the proposed stabilization, coordination, and navigation of MAVs is the visual relative localization based on the pattern detection by onboard cameras. The two main requirements, fast localization and onboard usability, require low computational demands for the image processing part. Therefore, we use an algorithm that allows for rapid detection and localization of simple circular patterns composed of concentric black and white circles of known diameter. Our algorithm (details described in [36]) outperforms common black-andwhite pattern detectors in terms of speed by an order of magnitude while achieving similar precision and robustness. An example of the localization pattern with a sketch of the possible operational space of the relative localization module is depicted in Fig. 1 .

The detection algorithm searches the image for circular patterns using a combination of flood-fill techniques, ondemand thresholding, and on-the-fly statistics calculation. The statistical information gathered on-the-fly is used to test whether the continuous areas of pixels are likely to represent the searched pattern, and quickly reject false candidates. The main advantage of the method is that it can be initiated from any position in the image without a performance penalty, which allows for a simple implementation of pattern tracking. In a typical situation, the algorithm processes only the area that is occupied by the pattern itself, which results in a significant performance boost.

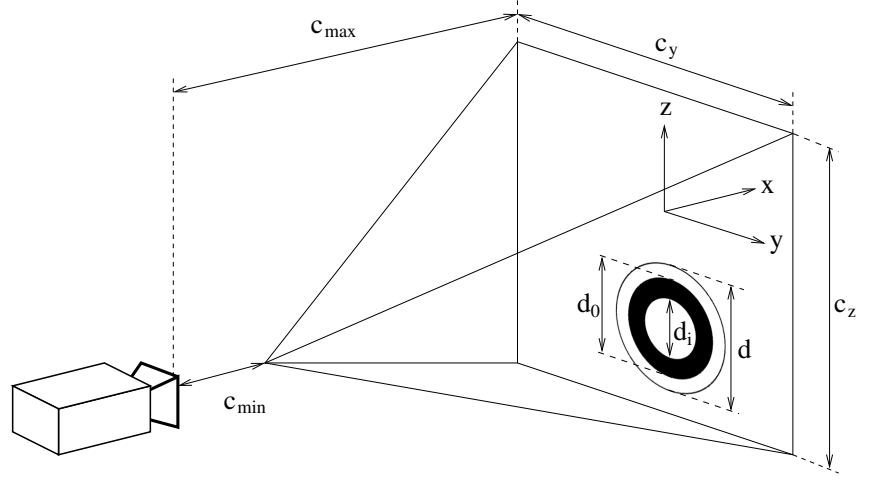

Fig. 1. The localization pattern and the operational space of the relative localization system.

In the initial phase of the pattern detection, the image is scanned for a continuous segment of black pixels. Segmentation of the pixels into black and white classes employs an adaptive thresholding that ensures good performance of the algorithm under variable light conditions, which is especially important in real-world outdoor experiments. Once a continuous segment of black pixels is found by the floodfill method, it is tested for minimum size and roundness. A pattern with outer and inner diameters $d_{o}, d_{i}$, bounding box dimensions $b_{u}, b_{v}$ and area $s$ is considered circular if its roundness $\rho_{\text {out }}$ is smaller than a predefined constant $\rho_{\max }$, i.e.

$$
\rho_{\text {max }}>\left|\rho_{\text {out }}\right|=\left|\frac{\pi}{4 s} b_{u} b_{v} \frac{d_{o}^{2}-d_{i}^{2}}{d_{o}^{2}}-1\right| .
$$

If a black region passes the roundness test, the flood-fill algorithm is initiated from the region's centroid in order to search for the inner white segment. Since the inner segments are circles and not rings, the roundness test for the inner white segments is simpler than (1):

$$
\rho_{\max }>\left|\rho_{i n}\right|=\left|\frac{\pi}{4 s} b_{u} b_{v}-1\right| \text {. }
$$

Then, the concentricity of segments and the ratio of their areas are tested. After passing these tests, the positions of the segments' pixels $u_{i}, v_{i}$ that were stored during the floodfill are used to calculate the ellipse center $u, v$ and covariance matrix $\mathbf{C}$ as follows:

$$
\mathbf{C}=\frac{1}{s} \sum_{i=0}^{s-1}\left(\begin{array}{ll}
u_{i} u_{i} & u_{i} v_{i} \\
u_{i} v_{i} & v_{i} v_{i}
\end{array}\right)-\left(\begin{array}{cc}
u u & u v \\
u v & v v
\end{array}\right)
$$

Note that $u_{i}, v_{i}$ are integers, and the computationally most expensive part of 3 is calculated using integer arithmetic.

Finally, the ellipse semiaxes $e_{0}, e_{1}$ are obtained from eigenvalues $\lambda_{0}, \lambda_{1}$ and eigenvectors $v_{0}, v_{1}$ of the covariance matrix $\mathbf{C}$ as follows:

$$
\begin{aligned}
& e_{0}=2 \lambda_{0}^{\frac{1}{2}} v_{0}, \\
& e_{1}=2 \lambda_{1}^{\frac{1}{2}} v_{1} .
\end{aligned}
$$

Knowing the length of the ellipse semiaxes, the final segment test is performed:

$$
\xi>\left|\pi e_{0} e_{1} s-1\right|
$$


The constant $\xi$ represents a tolerance value much lower than $\rho_{\max }$, because the ellipse dimensions $e_{0}, e_{1}$ are obtained from the covariance matrix with the sub-pixel precision. If the detected segments satisfy (4), they represent the localization pattern, and the obtained information is used to calculate the spatial dimensions of the pattern.

To obtain the relative distance of the pattern, we calculate the image coordinates of the ellipse (co-)vertices and transform these into canonical camera coordinates. This transformation takes into account not only the camera length and optical center, but also its radial distortion. The transformed vertices are then used to calculate the centre and axes of the ellipse in the canonical camera form. From the vertices, we calculate a conic $\mathbf{Q}$ such that the ellipse points $u^{\prime}, v^{\prime}$ satisfy

$$
\left(\begin{array}{c}
u^{\prime} \\
v^{\prime} \\
1
\end{array}\right)^{T} \mathbf{Q}\left(\begin{array}{c}
u^{\prime} \\
v^{\prime} \\
1
\end{array}\right)=0
$$

Then, we calculate the eigenvalues $\lambda_{0}, \lambda_{1}, \lambda_{2}$ and eigenvectors $q_{0}, q_{1}, q_{2}$ of the conic $\mathbf{Q}$ and use them to obtain the position of the pattern in space by the equations presented in [37]:

$$
\mathbf{x}=\frac{d_{o}}{\sqrt{-\lambda_{0} \lambda_{2}}}\left(q_{0} \lambda_{2} \sqrt{\frac{\lambda_{0}-\lambda_{1}}{\lambda_{0}-\lambda_{2}}}+q_{2} \lambda_{0} \sqrt{\frac{\lambda_{1}-\lambda_{2}}{\lambda_{0}-\lambda_{2}}}\right),
$$

where $d_{o}$ is the circular pattern diameter.

\section{A. Relative localization system performance}

The aim of this section is to show the performance of the relative localization system and to empirically specify its operational space. For details and experiments identifying the sensor model, see [38], [36]. Except the viewing angle, which can be clearly defined for each optical system (based on the lens), the most important factors that need to be considered in swarm stabilization and motion planning are the measurement accuracy and reliability. Both of these depend on the distance of the measured object, which provides a maximum measurable distance with acceptable system properties. The maximum measurable distance is then considered to be the range of the relative visual localization. This a priori obtained sensor model is crucial for the proposed multi-MAV motion planning and coordination. The detection reliability was measured with a pattern (with outer diameter $d_{o}=0.18$ m) placed on the camera optical axis at a distance $L$ from the camera and compared with the ground truth (see Table I).

Four different resolutions of the Caspa camera (used in all presented experiments) have been tested. The higher resolutions provide significantly better results, but at the cost of a decreasing measurement rate. The presented frame rates measured as Frames per Second (FPS) are obtained when the pattern is tracked (i.e. the blob is continuously detected without failures) ${ }^{4}$ If the pattern is not detected on the basis of its position in the previous image and the whole

\footnotetext{
${ }^{4}$ For the $320 \times 240$ resolution, the frame rate is limited by the camera, which can provide images at $60 \mathrm{~Hz}$.
}

picture needs to be processed, the frame rate sinks to 50$60 \%$ of the previous value. However, this lower value is not significant for the proposed control approach, since the measured relative distance is considered in the control loop only if the pattern is repeatedly detected. In the error and reliability data, we assume a systematic error proportional to the measured distance, which may be identified using the real distances and the Least Square Method (LSM). We present an average distance corrected by the systematic error (denoted as $\hat{L}$ ), since this value is more relevant for control and stability than the actual measured values of the distance in swarm applications. The corrected error in the distance estimate is obtained as $L_{e}=|L-\hat{L}|$, where $L$ is the ground truth. The standard deviation, $L_{\delta}$, presented as percentage of the measured distance, describes the repeatability of the measurements.

In addition to the variable resolution of the processed images, another aspect influencing the performance is the size of the pattern. As expected, with smaller patterns, the distance measurement error increases and the maximum measurable distance significantly decreases. For example, $480 \times 360$ image resolution allows the maxium measurable distances $L_{\max }=\{3.5,2.0,1.5,1.0,0.5\} \mathrm{m}$ with pattern diameters $d=\{18,9,8,7,5\} \mathrm{cm}$.

TABLE I

PERFORMANCE OF THE RELATIVE LOCALIZATION

\begin{tabular}{lcccccccc}
\hline resolution & \multicolumn{2}{c}{$\mathbf{3 2 0} \times \mathbf{2 4 0}$} & \multicolumn{2}{c}{$\mathbf{4 8 0} \times \mathbf{3 6 0}$} & \multicolumn{6}{c}{$\mathbf{6 4 0} \times \mathbf{4 8 0}$} & \multicolumn{2}{c}{$\mathbf{7 5 2} \times \mathbf{4 8 0}$} \\
\hline \hline$F P S$ & \multicolumn{2}{c}{60} & \multicolumn{2}{c}{46} & \multicolumn{2}{c}{30} & \multicolumn{2}{c}{27} \\
\hline \hline $\mathrm{L}$ & $L_{e}$ & $L_{\delta}$ & $L_{e}$ & $L_{\delta}$ & $L_{e}$ & $L_{\delta}$ & $L_{e}$ & $L_{\delta}$ \\
{$[\mathrm{m}]$} & {$[\mathrm{cm}]$} & {$[\%]$} & {$[\mathrm{cm}]$} & {$[\%]$} & {$[\mathrm{cm}]$} & {$[\%]$} & {$[\mathrm{cm}]$} & {$[\%]$} \\
\hline 0.5 & 1.2 & 0.1 & 0.9 & 0.4 & 3.6 & 0.6 & 4.3 & 1.2 \\
1.0 & 0.1 & 0.1 & 0.3 & 0.1 & 1.5 & 0.3 & 2.3 & 0.6 \\
1.5 & 0.6 & 0.1 & 0.9 & 0.1 & 0.1 & 0.1 & 0.4 & 0.5 \\
2.0 & 0.2 & 0.5 & 1.0 & 0.1 & 1.1 & 0.1 & 0.8 & 0.1 \\
2.5 & 2.0 & 0.2 & 0.0 & 0.2 & 0.7 & 0.2 & 1.8 & 0.2 \\
3.0 & 1.2 & 0.2 & 0.7 & 0.3 & 0.0 & 0.2 & 4.0 & 0.2 \\
3.2 & 3.0 & 0.2 & 1.8 & 0.7 & 3.5 & 0.2 & 2.3 & 0.2 \\
3.5 & - & - & 1.8 & 0.9 & 0.8 & 0.2 & 2.2 & 0.2 \\
4.0 & - & - & - & - & 5.4 & 0.4 & 3.3 & 0.4 \\
4.5 & - & - & - & - & 2.7 & 0.3 & 2.5 & 0.2 \\
5.0 & - & - & - & - & 2.4 & 0.6 & 3.4 & 0.6 \\
5.5 & - & - & - & - & 6.6 & 0.5 & 6.5 & 0.7 \\
\hline
\end{tabular}

In addition to this analysis, we conducted an experiment to evaluate the performance of the vision-based relative localization and to characterize its operational limits in flight conditions (see Fig. 2). During the experiment, two MAVs hovering in approximately static positions aim to localize the third MAV, which is following a predefined trajectory (see Fig. 3 for the ground truth positions of all MAVs obtained using VICON). All vehicles are equipped with cameras and identification patterns. This measurement was crucial for experimental evaluation of the limits of the space in which neighboring MAVs can be relatively localized. 


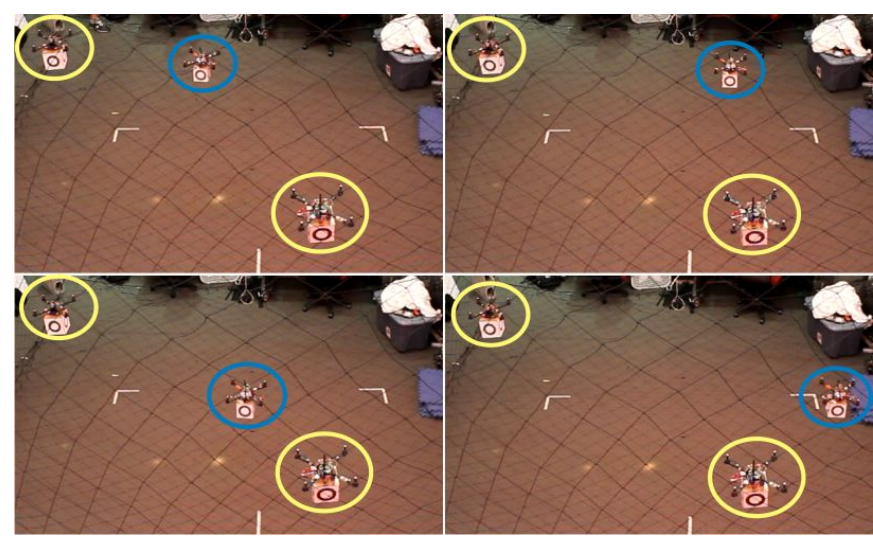

Fig. 2. Snapshots from measurements of the operational space of the visual relative localization system, which is important for specifying constraints for the planning of swarm movement.

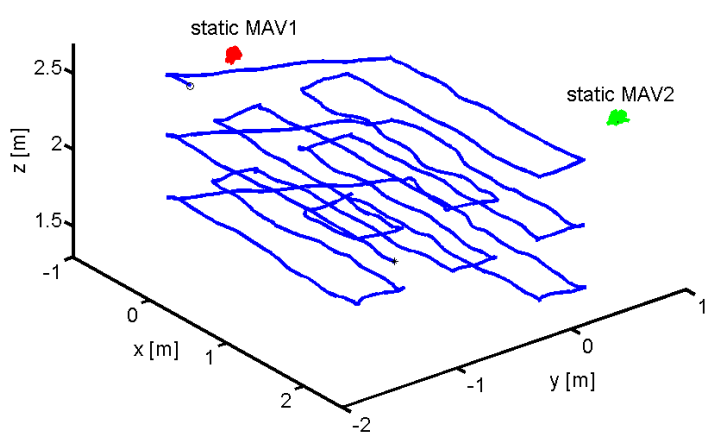

Fig. 3. Positions of MAVs captured by VICON during the experiment from Fig. 2

\section{MAV MODEL AND CONTROL SYSTEM}

\section{A. MAV model}

In the proposed approach, a suitable model of the quadrocopters is essential for use in simulations of MAVs movement, in motion planning, and in inter-vehicle coordination. This ensures that the motion constraints are satisfied during the planning process and that the obtained solution is feasible for the MAV-group. In this work, we rely on a simplified, decoupled dynamical model described as follows:

$$
\begin{aligned}
\ddot{x}^{W} & =\frac{U}{m}\left(\sin \psi^{I} \cos \phi^{W}-\sin \theta^{I} \sin \phi^{W}\right), \\
\ddot{y}^{W} & =\frac{U}{m}\left(\sin \theta^{I} \cos \phi^{W}+\sin \psi^{I} \sin \phi^{W}\right), \\
\ddot{z}^{W} & =\frac{U}{m} \cos \theta^{I} \cos \psi^{I}-g,
\end{aligned}
$$

where $\phi$ is the yaw angle, $\theta$ is the pitch angle, $\psi$ is the roll angle, $U$ is collective thrust, $m$ is the mass of the MAV, and $g$ is the gravitational acceleration. We consider 3 frames of reference (Fig. 4). The world frame $(W)$ that is fixed in the workspace, the body frame $(B)$ that coincides with particular MAV and the IMU frame $(I)$ in which the roll and pitch angles are measured.

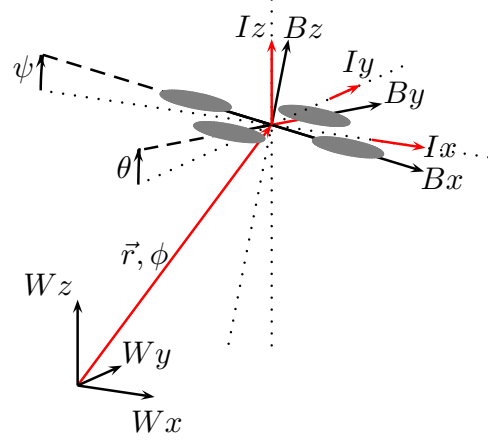

Fig. 4. The reference frames used in description of MAV control scheme W-world frame; B-body frame; I-IMU frame

\section{B. Control and stabilization scheme}

The complete system used for stabilizing the group members at desired relative distances (keeping the required shape of the group) and for motion simulation at the motion planning level is depicted in Fig. 5. The system consists of a controller (block $\mathrm{C}$ ), the stabilization unit $(\mathrm{S})$, and the model from (8). For deployment of the system, parameters of the linear model are identified using Least Squares Method from the measured flight data.

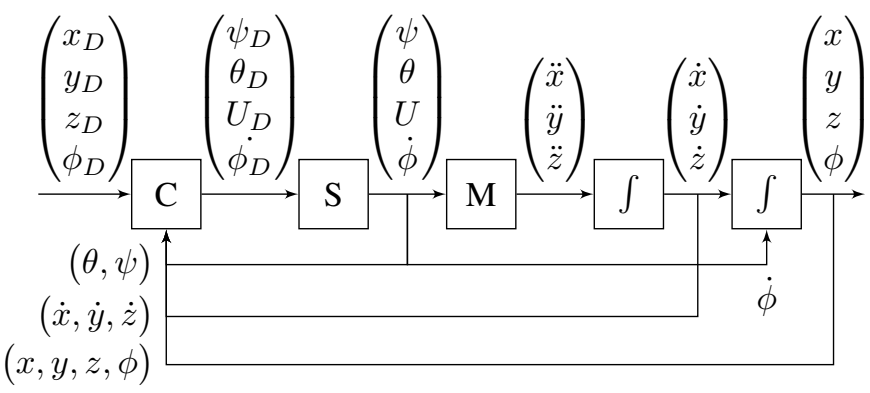

Fig. 5. Scheme of the system together with a controller. Position, its derivatives and $\phi$ are meant in the world frame, $\theta$ and $\psi$ in the IMU frame.

The flow of data within the proposed swarm stabilization system is shown in Fig. 6. The control scheme is suited for the MikroKopter quadcopter platform used for experimental evaluation of the visual relative localization based stabilization of the multi-MAV system in Section V The commercially available MikroKopter set includes a proprietary attitude stabilization board (Flight-CRTL) using an onboard Inertial Measurement Unit (IMU) for control feedback. The vision-based stabilization is built upon this lowest level and controls the relative positions between neighboring swarm entities. The solution is based on a custom board with the ATmega $\mu$-controller, which also serves as a communication hub between all onboard modules. Data received from the visual system, together with the output from IMU and from the PX4Flow smart camera sensor, serve as the control feedback at this level. The PX4Flow sensor provides information on the altitude and velocities relative 
to the surrounding environment. This setup is crucial for suppressing the motion oscillations within the group that are caused by the cumulative position error. The IMU provides angles $\theta^{I}, \psi^{I}$, PX4Flow provides $\dot{x}^{W}, \dot{y}^{W}$, and $z^{W}$, and the camera module provides relative coordinates to the particular neighbor $x_{n}^{I}, y_{n}^{I}, z_{n}^{I}$. The position controller computes the desired control outputs $\phi_{D}^{I}, \psi_{D}^{I}$, and $U_{D}^{I}$.

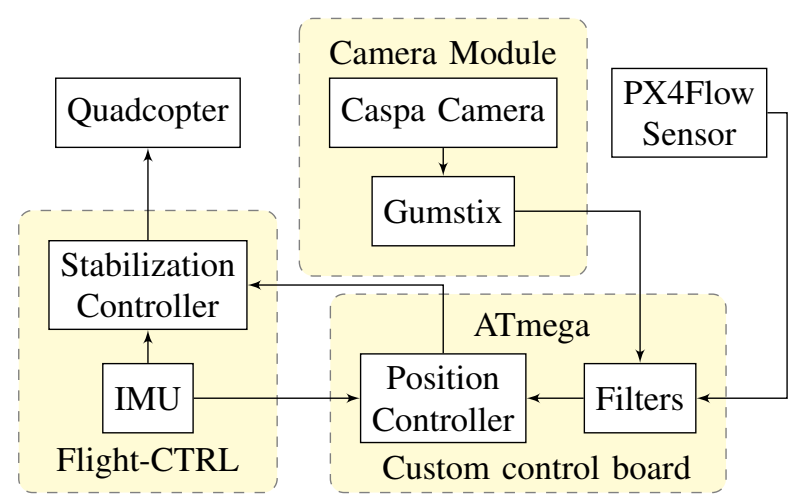

Fig. 6. Scheme of the data flow.

Three controllers are integrated in block $\mathrm{C}$; the forward and lateral controllers are identical due to the system decoupling. The following equation denotes the controllers outputs:

$$
\begin{aligned}
& \theta_{D}^{I}=K_{P} e_{x}+K_{D} \frac{d e_{x}}{d t}+K_{I} \int_{0}^{t} e_{x} \mathrm{~d} \tau \\
& \psi_{D}^{I}=K_{P} e_{y}+K_{D} \frac{d e_{y}}{d t}+K_{I} \int_{0}^{t} e_{y} \mathrm{~d} \tau \\
& U_{D}=L_{P} e_{z}+L_{D} \frac{d e_{z}}{d t}+L_{I} \int_{0}^{t} e_{z} \mathrm{~d} \tau
\end{aligned}
$$

where $K_{P}, K_{D}, K_{I}, K_{A}, L_{P}, L_{D}$, and $L_{I}$ denote the controller constants that need to be identified during the system setup. The control errors, $e_{x}^{I}, e_{y}^{I}$, and $e_{z}^{I}$, define the difference in the IMU coordinate system between the actual position of the controlled MAV and the desired position. The desired position is determined by the relative position to the circular pattern (resp. patterns) measured by the onboard visual localization, and by the desired relative position to the pattern (resp. patterns) given by a high-level planning method (see Section VI for examples of various planning approaches). The desired position may be dynamic in the sense of moving localization pattern (resp. patterns), which is placed on neighboring MAV (resp. MAVs), and/or in the sense of altering desired relative positions. In experiments with a static hovering MAV, the desired position is determined relatively to an initial position by the PX4Flow sensor.

\section{EXPERIMENTAL VERIFICATION OF THE SYSTEM WITH VISUAL RELATIVE LOCALIZATION IN CONTROL FEEDBACK}

In the first experiment, which was performed to demonstrate the performance of the control scheme, a single MAV is stabilized at a fixed relative distance to a static localization pattern. In this case, the MAV is also equipped with the localization pattern for its off-line global localization using an external fixed camera (see Fig. 7). The data from the external camera is used for experiment recording and offline analysis, while the MAV control relies on onboard sensors only. The results from this external camera are plotted in Fig. 8. The mean control deviation from the desired equilibrium was $0.11 \mathrm{~m}, 0.12 \mathrm{~m}$, and $0.04 \mathrm{~m}$ with standard deviations $0.14 \mathrm{~m}, 0.15 \mathrm{~m}$, and $0.05 \mathrm{~m}$, measured in the $x, y$, and $z$ coordinates. The slight motion oscillations are caused mainly by the noise in the sensor data. See Fig. 9 for data from the camera module with Gumstix, optical flow obtained from the PX4Flow sensor, and the outputs of the designed controllers. A detailed view of a sample from Fig. 9 is presented in Fig. 10

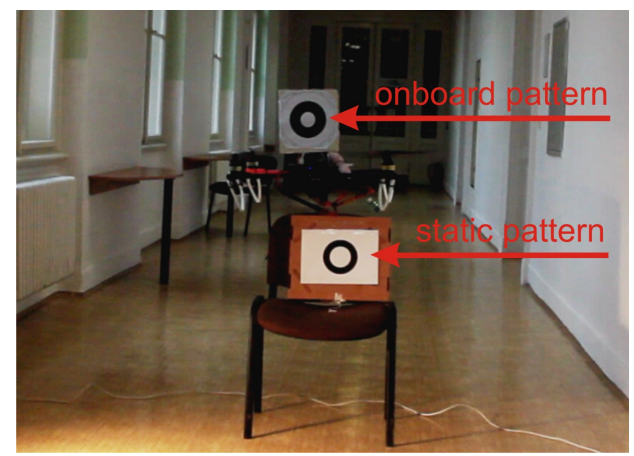

Fig. 7. MAV stabilized at a fixed relative distance to the static pattern. The onboard pattern is used for external localization, which gives the groundtruth for experiment evaluation.

The stabilization of neighboring vehicles with a predefined mutual distance is shown in the outdoor experiment in a former pit mine (see Fig. 11 for pictures from the experiment). The experiment verifies the ability of the system to follow a moving "leader" MAV with an attached localization pattern. The first MAV (the leader) is controlled along a pre-planned trajectory based on the visual odometry from a PX4Flow sensor (the pose estimate is obtained by integrating the optical flow from the down-looking camera). The second MAV follows the first one at a fixed desired spacing based on feedback from the onboard visual relative localization system. The elevation above the slope of the mine is fixed for both MAVs based on the feedback from the sonar.

The same experiment was repeated in the presence of wind on a flat road. We demonstrated that the system is also able to stabilize the formation with minimal influence of the wind on the performance of the system (see Fig. 12 for pictures taken during the experiment). Videos of these experiments can be downloaded from [39].

The aim of the experiment presented in Fig. 13 is to demonstrate flying in strings of the relatively stabilized MAVs using the proposed system. In the case of stabilization of large groups of MAVs, it is difficult to ensure that all MAVs are stabilized directly to the same MAV (a common leader). Naturally, more complex networks arise in swarms or formations of MAVs, in which always some robots need to be stabilized relatively to neighbors that are already stabilized 


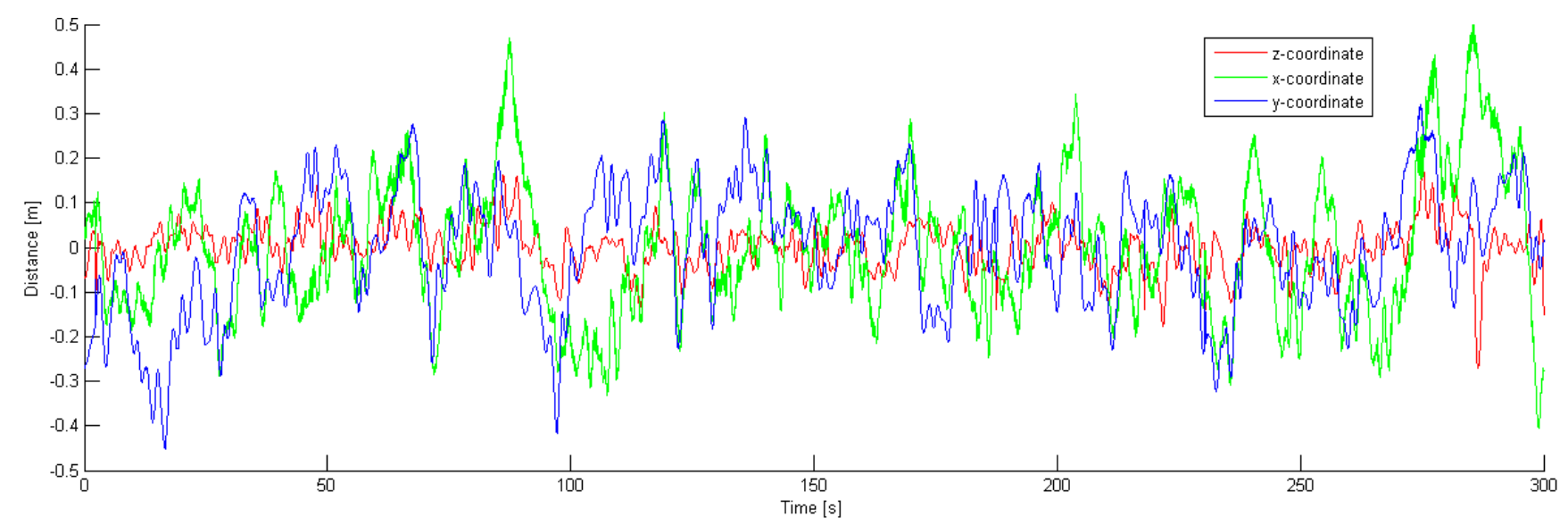

Fig. 8. Deviation from the desired equilibrium located at $2.5 \mathrm{~m}$ from the static circular pattern (experiment in Fig. 7). Data obtained from the record of the external camera.
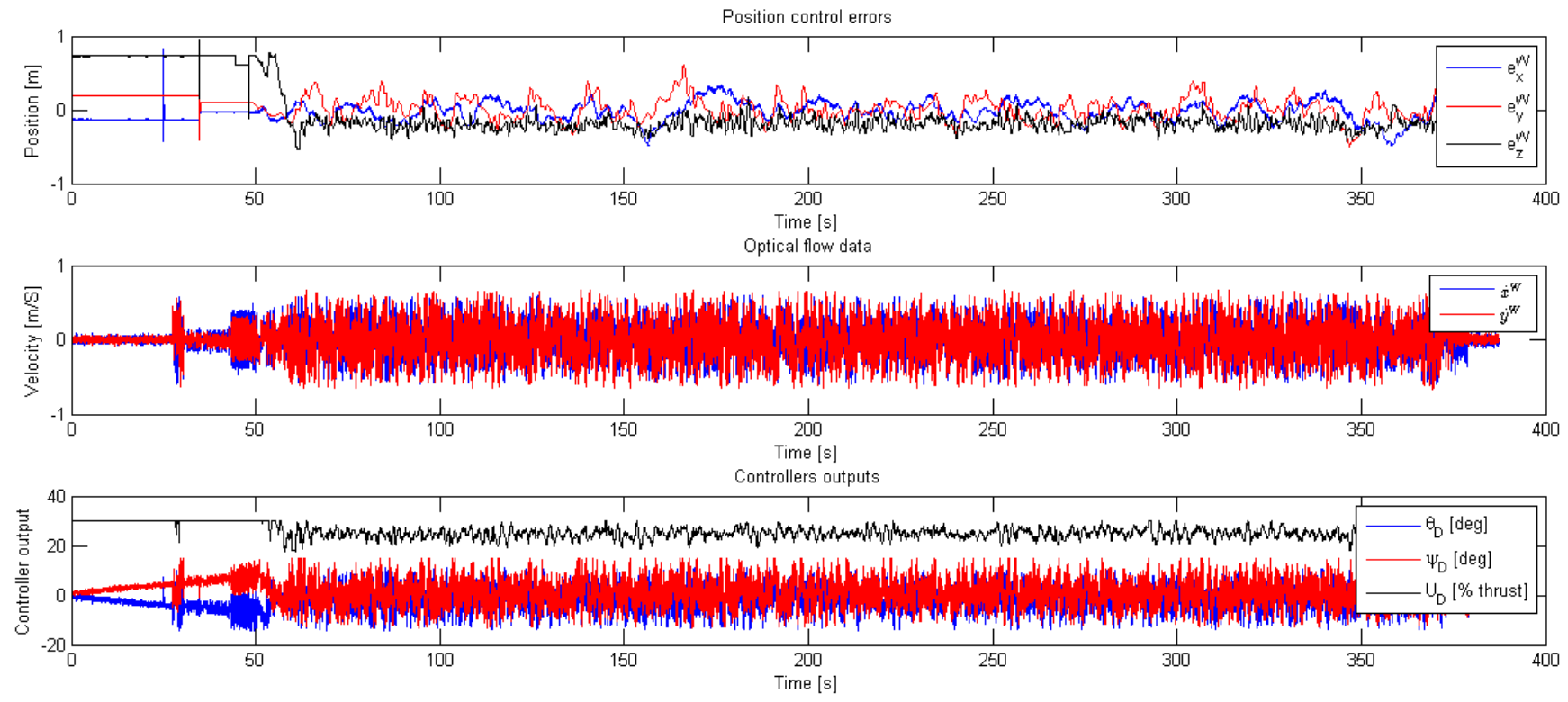

Fig. 9. Sensor data and controller output during the experiment shown in Fig. 7 The first picture presents the output (in $x, y$, and $z$ coordinates) from the onboard relative localization module. The output from the PX4Flow sensor is shown in the second picture, while the outputs of the controllers are presented in the third plot.

relatively to another robot, etc.

Data in Table $\mathrm{II}$ with results of experiments from Fig. 13 show only a slight increase of motion oscillation of an MAV following another MAV in a comparison with the situation where the same MAV is stabilized relatively to a static pattern. The moving pattern introduces additional noise into the measurement of the relative localization and furthermore slightly decreases performance of the vision system as described in [1]. See the second and the fourth columns of the table that show flight performance of the MAV with id 3 in two different roles: 1) as a leader stabilized relatively to the static pattern, 2) as a follower stabilized relatively to another leader. Similar comparison can be seen in the third and the sixth columns for the MAV with id 2.

In addition, the motion oscillations of the MAV with id 3 are comparable in the MAV pair experiment (fourth column of the table), where the MAV with id 3 acts as the first follower that is directly stabilized to the leader, and in the 3 MAVs in line experiment (seventh column), where the MAV with id 3 acts as the second follower that is indirectly stabilized to the leader; over another follower. Taking into consideration the data that describes the motion performance of the MAVs with id 2 and 3, one can see that the difference between these two robots is more significant than the difference between motion of MAV 3 in roles of the follower 1 and 2. Even though all MAVs are controlled by the same systems with the same parameters and sensors, the small differences during their manufacturing influence the flight performance. The independence of the control performance from the number of robots in the controlled 

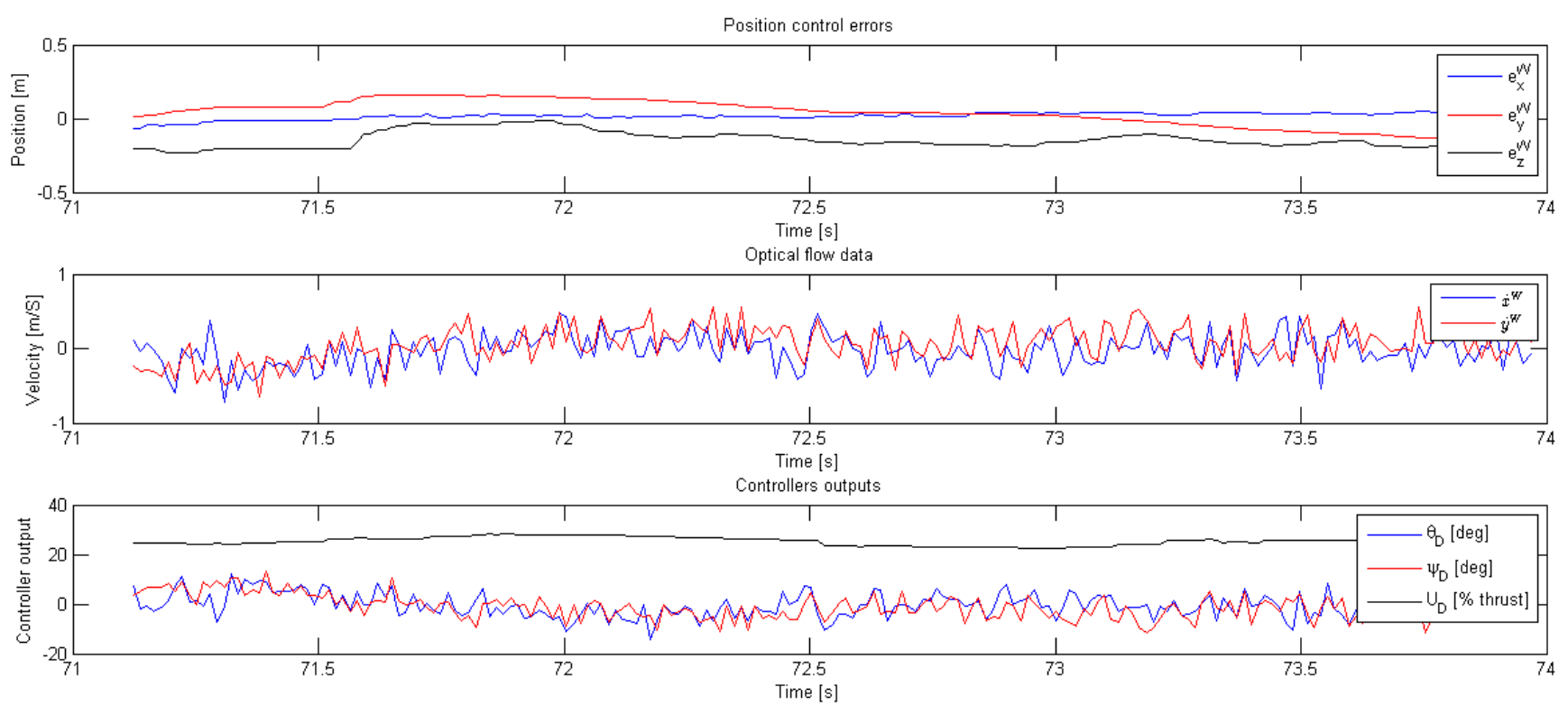

Fig. 10. Zoomed view on data from Fig. 9

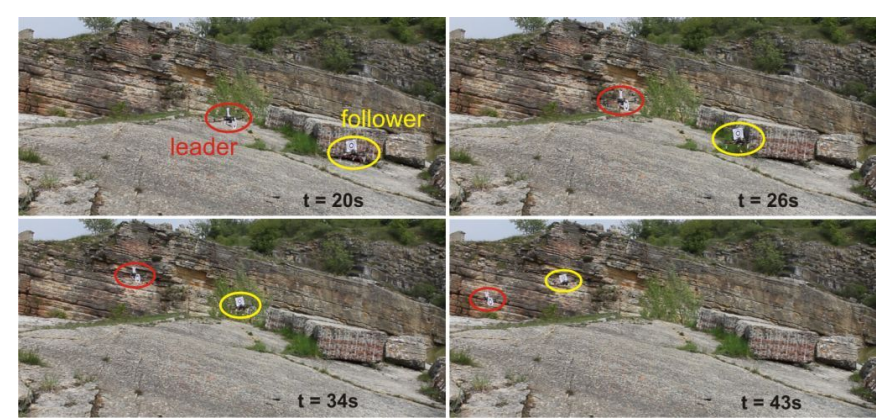

Fig. 11. Leader-follower formation flying on a slope surface.

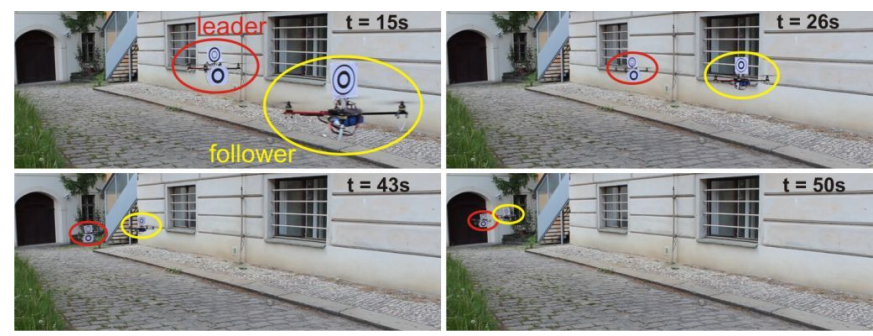

Fig. 12. Leader-follower formation in windy conditions.

string was observed also in case of moving relatively stabilized formations (see Fig. 11 and 12), which is crucial in most of the applications requiring the group stability. In all experiments in Fig. 13, the relative distance between neighbouring MAVs and between the MAV and the static pattern was $2.5 \mathrm{~m}$.

In addition to these outdoor experiments, the vision system was tested in numerous experiments of various multi-robot applications with the VICON motion capture system as a reference. The precision and reliability of the external motion
TABLE II

TESTS OF THE FLIGHT PERFORMANCE OF MAVS IN A STATIC PLATOON-LIKE FORMATION HOVERING ON A SPOT. SNAPSHOTS FROM THE EXPERIMENTS ARE SHOWN IN FIG.13 AND VIDEOS ARE AVAILABLE AT [39]. THE MEAN ERROR AND STANDARD DEVIATION ARE MEASURED FROM A FIXED EQUILIBRIUM BY THE EXTERNAL VIDEO SYSTEM [36]. THE EQUILIBRIUM IS DEFINED BY A FIXED RELATIVE POSITION TO THE ONBOARD PATTERN IN CASE OF FOLLOWERS, BY A FIXED RELATIVE DISTANCE TO THE STATIC PATTERN IN CASE OF THE LEADER IN THE

1-MAV AND MAV PAIR EXPERIMENTS, OR THE EQUILIBRIUM IS SET AS A FiXed INITIAL POSITION IN CASE OF THE LEADER IN THE 3 MAVS IN LINE EXPERIMENT. THE RELATIVE DISTANCE BETWEEN NEIGHBOURING MAVS AND BETWEEN THE MAV AND THE STATIC PATTERN WAS $2.5 \mathrm{M}$

IN ALL EXPERIMENTS. THE STATISTICS WERE OBTAINED FROM APPROXIMATELY 900 SAMPLES FOR EACH OF THE EXPERIMENTS. APPROXIMATELY 30S LONG RECORDS WITH THE RATE 30 FRAMES PER SECONDS WERE ANALYSED FOR EACH EXPERIMENTAL FLIGHT.

\begin{tabular}{lcccccc}
\hline exp. type & 1-MAV & \multicolumn{2}{c}{ MAV pair } & \multicolumn{3}{c}{ 3 MAVs in line } \\
\hline \hline MAV id & 3 & 2 & 3 & 1 & 2 & 3 \\
MAV role & L & L & foll. 1 & L & foll. 1 & foll. 2 \\
\hline mean error [cm] & 13.1 & 19.6 & 14.6 & 14.7 & 20.0 & 14.9 \\
stand. dev. [cm] & 6.9 & 11.2 & 8.2 & 7.8 & 12.5 & 8.3 \\
\hline
\end{tabular}

capture system is sufficient to consider the obtained data as the ground truth [40]. Details on the tested multirobot scenarios and the obtained results are given in Section VI. In the formation driving experiments (Fig. 16 21), where the relative distance between quadrocopters is almost constant, the reliability of the measurement is approximately $98 \%$. If we exclude the outliers caused by identification of a "wrong" $\mathrm{MAV}$, or by occlusions, the mean error of the relative distance is $1.1 \mathrm{~cm}$ (with standard deviation $0.9 \mathrm{~cm}$ ) at a distance of $1.5 \mathrm{~m}$ between neighboring MAVs. The maximum error is 


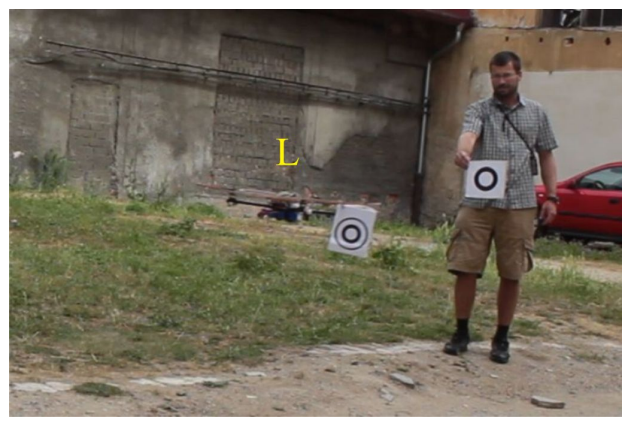

(a) 1-MAV. MAV relatively stabilized to a static pattern using the visual relative localization in control feedback.

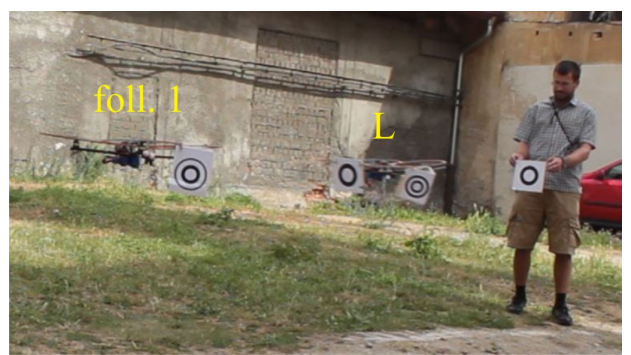

(b) MAV pair. The MAV on the right (follower 1) is relatively stabilized to a static pattern. The second MAV (follower 2) is relatively stabilized to the follower 1 .

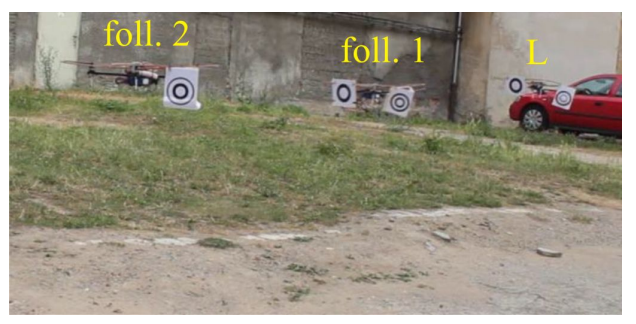

(c) 3 MAVs in line. The MAV in the most right (leader) hovers on spot using the PX4Flow sensor. The middle MAV (follower 1) is relatively stabilized to the leader. The MAV in the most left (follower 2) is relatively stabilized to the follower 1 .

Fig. 13. Verification of the proposed approach for stabilization of MAVgroups based on the visual relative localization. Experiments show (see data in Table II that due to the measured relative speed between the MAVs and surface, which is employed in control feedback, position oscillations do not increase with the size of the group being relatively stabilized.

always less than $4 \mathrm{~cm}$ in these experiments. In the swarm experiments (Fig. 24 25 and 28|30, the relative distance between quadrocopters differs from $1 \mathrm{~m}-2.5 \mathrm{~m}$ and the measurement reliability is approximately $95 \%$. The mean error of the relative distance is $1.3 \mathrm{~cm}$ (with a standard deviation of $1.7 \mathrm{~cm}$ ). This slightly worse performance is caused by a longer relative distance between the robots and by their relative motion, which may spoil some pictures captured by the onboard cameras (the camera and the objects in the images move independently). The frame rate exceeds 30 frames per second if the images are processed online and pictures are not stored in the memory of the camera module. If unprocessed images need to be stored for a later evaluation of the experiment, the rate has to be reduced to $10-15 \mathrm{fps}$.

\section{Multi-ROBOT SCENARIOS DEMONSTRATING THE PRACTICAL USABILITY OF THE SYSTEM}

The aim of this section is to present examples of practical utilization of the proposed system. We have chosen three general approaches to MAV deployment that are currently solved in state-of-the-art literature (leader-follower formation flying, swarm-inspired stabilization, and multi-MAV surveillance) to show how these scenarios can be solved using the proposed system. Each of the multi-robot scenarios proposes a different approach to motion planning and coordination of the MAV-group. The common challenge lies in the necessity to satisfy the MAV motion constraints and the constraints imposed by the relative localization. Therefore, the description of these methods is focused on integration of the relative localization constraints into the planning algorithms. In addition, in the experimental parts of this section, the tests with the VICON motion capture system in control feedback verify that the trajectories of the MAVs obtained by the proposed high-level planning systems are feasible for real MAV-groups. This means that the obtained trajectories respect the MAV dynamics, the localization constraints, and the environment constraints (obstacles and no-fly zones). Besides, the aim of these experiments is to evaluate the performance of the camera module and the localization algorithm in multi-MAV applications. The results of the experiments are compared with the ground truth and are attached to the description of each method in the following subsections.

\section{A. Scenario 1: Leader-follower formation flight.}

In this scenario, a formation of multiple MAVs reaches a desired target region in a complex environment with obstacles, while maintaining predefined relative positions. The desired shape of the formation can be temporarily changed only if it is enforced by environmental constraints (e.g. in narrow passages). The proposed formation control mechanism is suited for the real-world deployment of autonomous robots relying on the onboard visual relative localization, which brings additional movement constraints to the MAV team. The method is based on a leader-follower technique, where the team of robots is stabilized by sharing knowledge of the leader's position within the formation (see the original leader-follower approach [41] designed for a group of ground robots (UGVs) and the extension of the leader-follower approach for heterogenous MAVs-UGVs teams in [42], [43] for details). The method presented in this section is an extension of our work introduced in conference paper [44], where only simulation results were presented and where the requirements on the onboard relative localization necessary for the HW experiments, which is the main contribution of this paper, were not included.

We do not rely on following a given trajectory, as in most of the state-of-the-art methods [45], [46], [47]. We propose to integrate the stabilization of followers in the desired positions behind the leader together with the trajectory planning into 


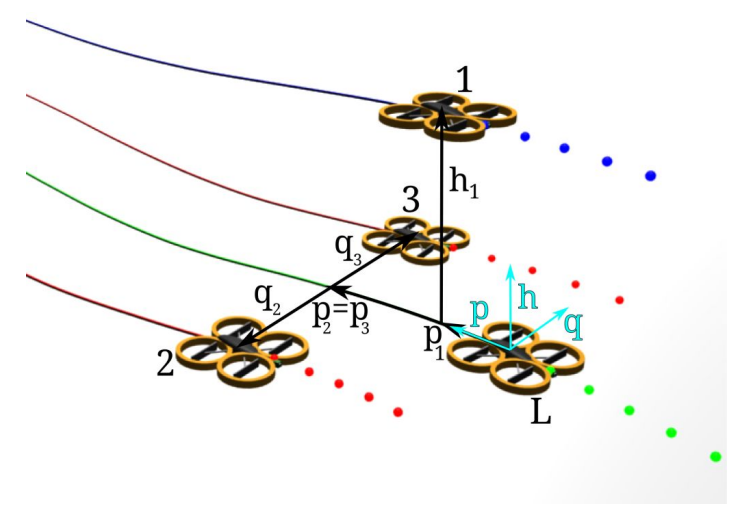

Fig. 14. The desired shape of the formation described in curvilinear coordinates.

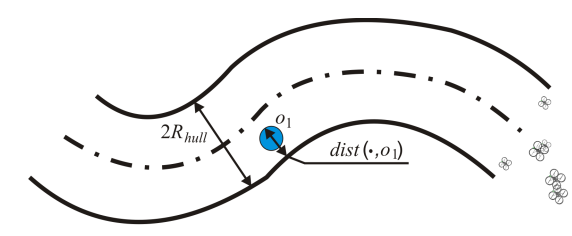

(a) Contours of the convex hull projected along the leader's trajectory. An obstacle is denoted inside the hull to clarify the meaning of function $\operatorname{dist}\left(X_{L}(\cdot), o_{l}\right)$.

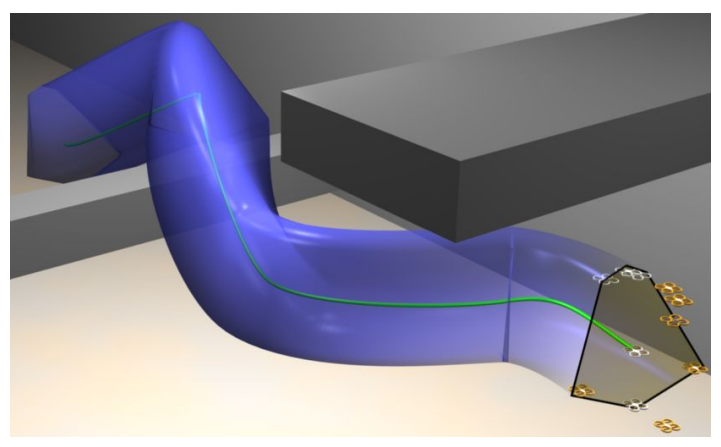

(b) $3 \mathrm{D}$ visualization of the convex hull projected along the leader's trajectory (the circular obstacle is hidden inside the hull).

Fig. 15. An example of the dilated convex hull projected along a trajectory. This trajectory would be infeasible for the formation stabilized by the presented approach, since an obstacle appears inside the convex hull.

a desired goal area with obstacle avoidance ability for the entire formation. The global trajectory planning is directly integrated into the formation control mechanism, which is important for finding a feasible solution for the proposed approach using the relative visual localization of the team members. For stabilization of the MAV group via the onboard relative localization, it is crucial that direct visibility between team members is not interrupted by an obstacle. Thus, in the trajectory planning process, direct visibility is ensured by penalizing collisions between obstacles and a 2D convex hull of the positions of followers, which represents the $3 \mathrm{D}$ formation. The $2 \mathrm{D}$ convex hull is obtained as a projection of positions of followers into a plane that is orthogonal to the trajectory of the virtual leader in its current position (see Fig. 15(a)). For the obstacle avoidance function described in eq. (11), the convex hull is dilated by a safety radius, which is considered around each MAV, to keep obstacles at a desired distance from the followers. The trajectory planning into the desired goal region and the immediate control of the formation is then integrated in a single optimization process with this obstacle avoidance function. The method can continuously respond to changes in the vicinity, while keeping the cohesion of the immediate control inputs with the directions of movement of the MAV formation in the future.

In the algorithm, followers follow the trajectory of the leader at distances defined in the $p, q, h$ curvilinear coordinate system, as visualized in Fig. 14. The position of each follower $i$ is uniquely determined: 1$)$ by states $x_{L}\left(t_{p_{i}}\right)$ in the traveled distance $p_{i}$ from the actual position of the leader along its trajectory, 2) by the offset distance $q_{i}$ from the leader's trajectory in the perpendicular direction and, 3) by the elevation $h_{i}$ above the leader's trajectory, as follows:

$$
\begin{aligned}
& x_{i}(t)=x_{L}\left(t_{p_{i}}\right)+\left(-q_{i} \sin \left(\theta_{L}\left(t_{p_{i}}\right)\right), q_{i} \cos \left(\theta_{L}\left(t_{p_{i}}\right)\right), h_{i}\right)^{T} \\
& \theta_{i}(t)=\theta_{L}\left(t_{p_{i}}\right)
\end{aligned}
$$

where $x_{L}\left(t_{p_{i}}\right)$ is the position of the leader at the time when the virtual leader was at traveled distance $p_{i}$ behind the current position and $\theta_{L}$ is the yaw of the leader at time $t_{p_{i}}$.

The short-term trajectory planning responding to the local workspace of the robots and the long-term trajectory planning providing a plan to the target location are solved together in a single optimization step. The leader's trajectory encoded into a vector of constant control inputs at time $t$ is used as the optimization vector $X_{L}(t)=\left[\nu_{L, 1}, v_{L, 1}\right.$, $k_{L, 1}, \ldots, \nu_{L, N}, v_{L, N}, k_{L, N}, \nu_{L, N+1}, v_{L, N+1}, k_{L, N+1}$, $\left.\delta_{L, N+1}, \ldots, \nu_{L, N+M}, v_{L, N+M}, k_{L, N+M}, \delta_{L, N+M}\right]$ to include both, the local and the global trajectory planning. The vector $X_{L}(\cdot)$ consists of the normal velocity $\nu_{L, \cdot}\left[\mathrm{m} \cdot \mathrm{s}^{-1}\right]$, the tangential velocity $v_{L,} \cdot\left[\mathrm{m} \cdot \mathrm{s}^{-1}\right]$, the curvature $k_{L,} \cdot\left[\mathrm{m}^{-1}\right]$, and the length of the time interval $\delta_{L,} \cdot[\mathrm{s}]$. The curvature $k_{L \text {, }}$. of the trajectory followed by the leader is constant within each control segment and may vary along the whole trajectory. The time interval $\delta_{L, j}$ is constant if $j \in\{1 \ldots N\}$ and becomes variable if $j \in\{N+1 \ldots N+M\}$. The constant time interval is denoted as $\Delta t$ and is set as $\delta_{L, j}:=$ $\Delta t=0.1 s, j \in\{1 \ldots N\}$, in the experiments. $N$ is the number of transition points in the short control horizon with the constant $\Delta t$ between the transition points. $M$ is the number of transition points in the long planning horizon with variable $\delta_{L, j}$ between the transition points. The trajectory is obtained from the optimization vector by applying the constant control inputs into the model in Section IV.

The leader's control problem with the obstacle avoidance ability can then be transformed to minimization of the multi- 


$$
\begin{aligned}
& F_{L}\left(X_{L}(\cdot)\right)= \\
& \sum_{l=1}^{n_{o}}\left(\min \left\{0, \frac{\operatorname{dist}\left(X_{L}(\cdot), o_{l}\right)}{\operatorname{dist}\left(X_{L}(\cdot), o_{l}\right)-R_{\text {hull }}}\right\}\right)^{2}+\alpha \sum_{j=N+1}^{N+M} \delta_{L, j} .
\end{aligned}
$$

The first part of the function prevents the formation from colliding with obstacles. The number of considered obstacles is denoted as $n_{o}$, and $o_{l}$ denotes the $l$-th obstacle. Its value is zero if all obstacles are outside the projected convex hull, which is formed by MAVs following the leader in their desired positions within the formation. $R_{h u l l}$ is the radius of the convex hull depicted in Fig. 15(a). The value goes to infinity as an obstacle approaches into the center of the hull. This ensures that direct visibility between the robots will not be broken by an obstacle located among them. The value of the second term is based on an estimation of the total time to reach the desired target region, which must be minimized. The influence of the obstacle avoidance function and the endeavour of the trajectory planning to reach the target region in minimum time are weighted by constant $\alpha$.

To ensure feasibility of the obtained solution, the optimization process is subject to a set of constraints. The first constraint, which is necessary for a convergence of the formation driving process into the desired equilibrium (the desired target region), requires that the final transition point of the planning horizon is inside the target region. In addition, control inputs have to be constrained since the planning approach for the leader must respect the constraints given by mechanical capabilities of all followers. The admissible control set for the leader can be determined by applying the leader-follower approach for $i=1, \ldots, n_{r}$ as $k_{L, \max }=$ $\min \left(k_{i, \max } /\left(1+q_{i} k_{i, \max }\right)\right), k_{L, \min }=\max \left(-k_{i, \max } /(1-\right.$ $\left.\left.q_{i} k_{i, \max }\right)\right), v_{L, \max }=\min \left(v_{i, \max } /\left(1+q_{i} k_{L}\right)\right), v_{L, \min }=$ $\max \left(v_{i, \min }\left(1+q_{i} k_{L}\right)\right), \nu_{L, \max }=\min \left(\nu_{i, \max }\right), \nu_{L, \min }=$ $\max \left(\nu_{i, \min }\right)$, where $k_{i, \max }, v_{i, \max }, v_{i, \min }, \nu_{i, \max }$ and $\nu_{i, \min }$ are limits on the control inputs of the $i$-th follower. These restrictions must be applied to satisfy different values for the curvature and the speed of the robots in different positions within the formation. For example, the robot following the inner track during turning goes more slowly but with a bigger curvature than the robot further from the center of the turning, due to the fact that the followers turn around the same Instantaneous Center of Curvature (ICC) and at the same angular speed.

The states specified by the trajectory of the leader of the formation obtained as a result of the optimization are transformed for the followers using the transformation in eq. (10). These desired states are used for the trajectory tracking algorithm with the obstacle avoidance function, which enables responses to events that occur in the environment behind the actual position of the leader. The trajectory is encoded into a vector of constant control inputs and it is used as the optimization vector $X_{i}(\cdot)=\left[\nu_{i, 1}, v_{i, 1}, k_{i, 1}, \ldots, \nu_{i, N}, \tau_{i, N}, k_{i, N}\right]$ for the $i$-th follower. For the motion planning of the follow-
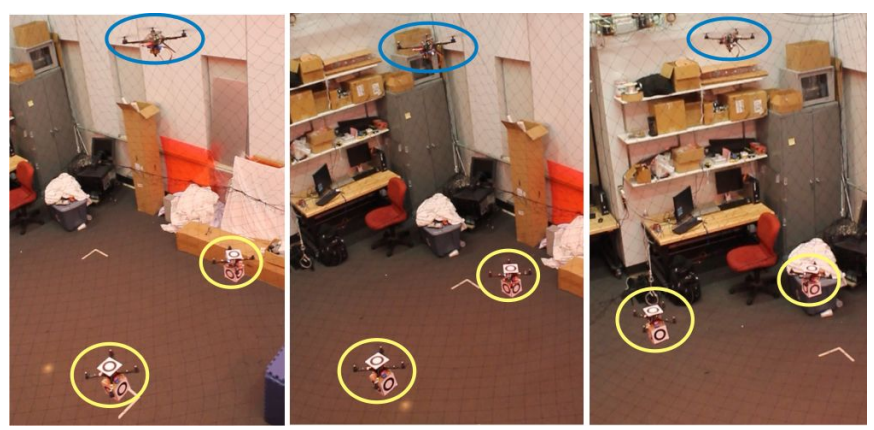

Fig. 16. Experiment with a triangular formation of 3 MAVs.

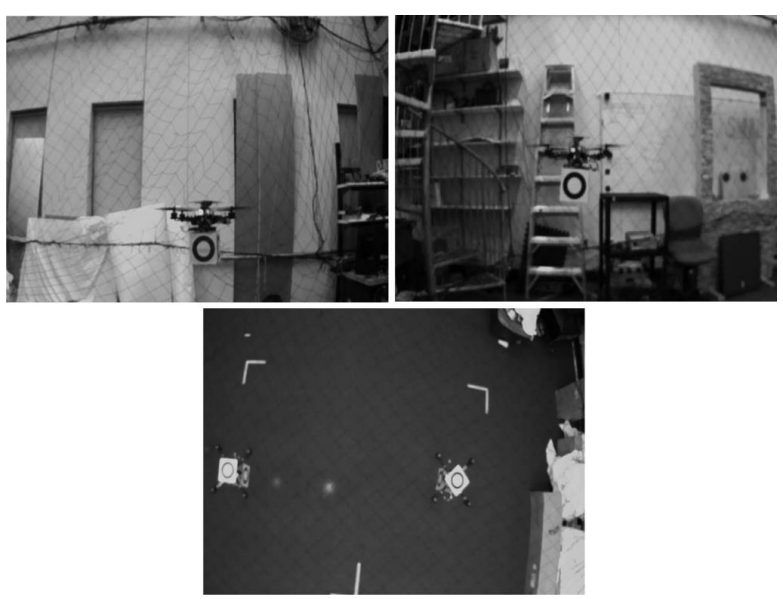

Fig. 17. Examples of pictures obtained by the onboard cameras for the relative localization (exp. in Fig 16 .

ers, only the short-term horizon with a constant sampling time is employed. The discrete-time trajectory tracking for each follower is transformed to minimization of the multiobjective cost function $F_{i}\left(X_{i}(\cdot), X_{L}\right)$ subject to set of constraints as follows:

$$
\begin{aligned}
F_{i}\left(X_{i}(\cdot), X_{L}\right)= & \\
& \sum_{l=1}^{n_{o}}\left(\min \left\{0, \frac{\operatorname{dist}\left(X_{i}(\cdot), o_{l}\right)-r_{s}}{\operatorname{dist}\left(X_{i}(\cdot), o_{l}\right)-r_{a}}\right\}\right)^{2}+ \\
& \sum_{j \in \bar{n}_{n}}\left(\min \left\{0, \frac{d_{i, j}\left(X_{i}(\cdot), X_{j}\right)-r_{s}}{d_{i, j}\left(X_{i}(\cdot), X_{j}\right)-r_{a}}\right\}\right)^{2}+ \\
& \beta\left(\sum_{j=1}^{N}\left|{ }^{d} x_{i, j}-x_{j}\right|^{2}+\sum_{j=1}^{N}\left({ }^{d} \theta_{i, j}-\theta_{j}\right)^{2}\right) .
\end{aligned}
$$

The first sum penalises solutions with a distance to an obstacle less than the detection radius $r_{s}$. The penalty function goes to infinity as an obstacle approaches a distance equal to the avoidance radius $r_{a}$. If the distance between an obstacle and the trajectory is less than $r_{a}$, the solution is considered infeasible (the obstacle proximity constraint of the optimization is violated). In the second sum of the cost function, the other members of the team are considered as dynamic obstacles in case of an unexpected behavior of 

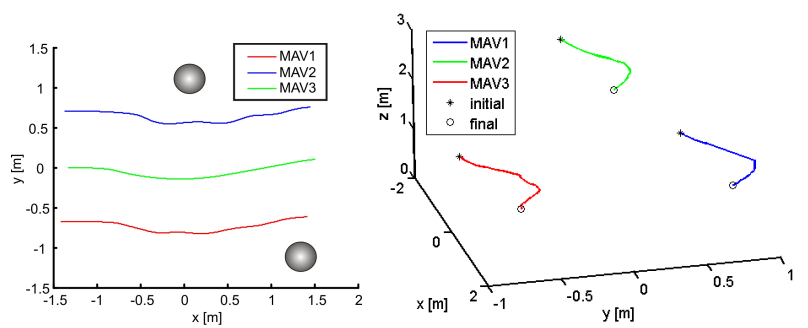

Fig. 18. Trajectories of MAVs in the experiment from Fig. 16 recorded by the VICON system. Positions of the virtual obstacles are denoted by the circles.

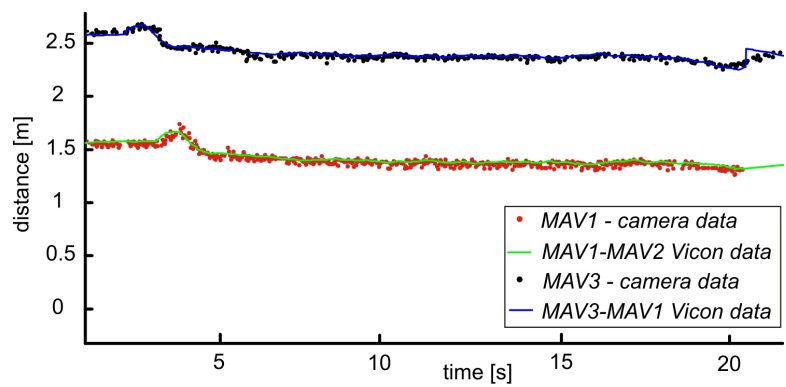

Fig. 19. Relative distances between MAV1-MAV2 and MAV3-MAV1 in the experiment from Fig. 16 The dots correspond to raw data obtained from the visual relative localization, and the curves are reference values provided by the VICON motion capture system.

defective neighbors deviating from their desired positions within the formation. Function $d_{i, j}\left(X_{i}(\cdot), X_{j}\right)$ returns the minimal distance between the planned trajectory of follower $i$ and the plan of other followers $j \in \bar{n}_{n}$, where $\bar{n}_{n}=$ $\left\{1, \ldots, i-1, i+1, \ldots, n_{r}\right\}$.

The last term of the cost function penalizes a growing Euclidean distance between the desired positions ${ }^{d} x_{i, j}, j \in$ $\{1 \ldots N\}$, obtained from the actual leader's trajectory $X_{L}$, and the positions of the $i$-th follower. Also the differences between the desired yaw angles ${ }^{d} \phi_{i, j}, j \in\{1 \ldots N\}$, and the actual yaw of follower $i$ are penalized. The influences of the obstacle avoidance function and the trajectory following term are weighted by constant $\beta$. Values $\alpha=1$ and $\beta=1$ were used in all experiments in this article, but the approach does not require fine tuning of these parameters and the same values can be efficiently used in different scenarios.

In addition to the constraint, which is satisfied if the distance between the trajectory corresponding to the particular solution of the optimization and all obstacles is greater than $r_{a}$, the control inputs are constrained to satisfy the motion constraints of the employed MAVs (limits on forward and ascending velocities etc.).

1) Experimental evaluation of the planning technique in flight conditions: In this section, the feasibility of results of the formation planning approach is verified by experiments with multiple MAVs. Two virtual obstacles, the no-fly zones depicted in Fig. 18, are considered in the workspace to demonstrate the obstacle avoidance ability. Three MAVs equipped with the visual relative localization modules [38] are stabilized in triangular and line formations. In the tri-
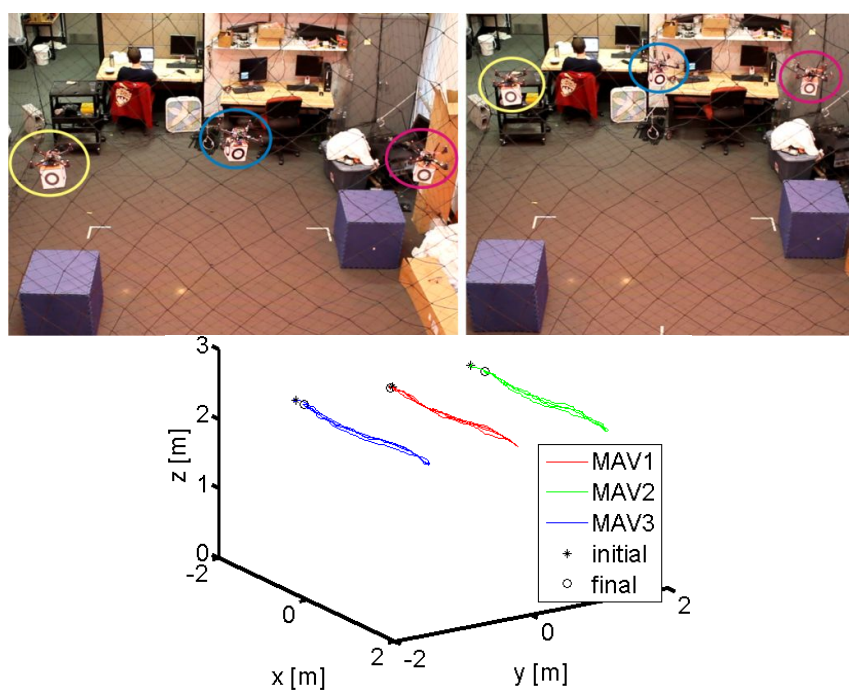

Fig. 20. Experiment with the line formation of 3 MAVs.
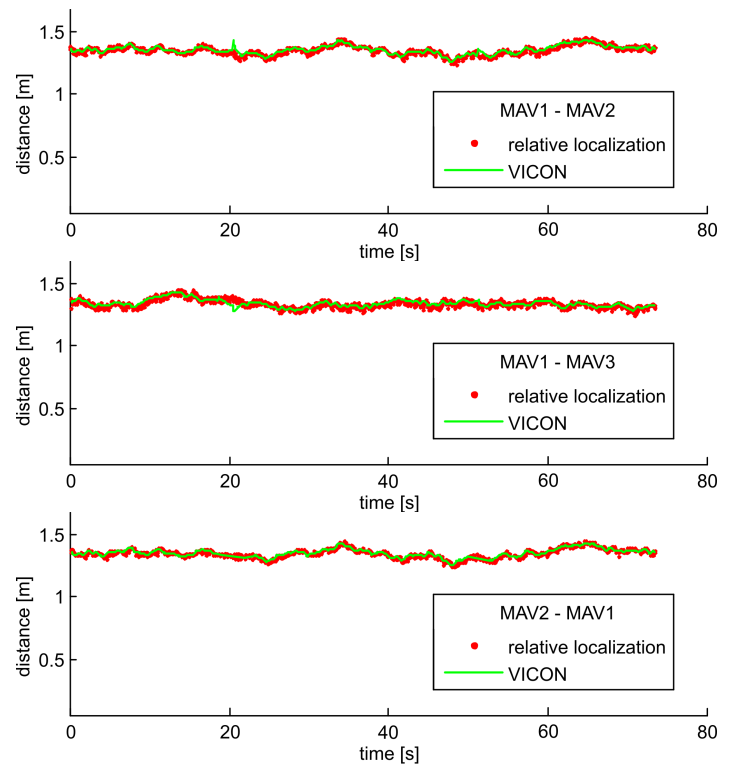

Fig. 21. Relative distances between MAVs obtained by the onboard cameras for formation stabilization. VICON data record is plotted as a reference.

angular formation (see Fig. 16]19, an MAV with a camera pointed down is flying above two other MAVs with side looking cameras. The experiment with the line formation of three MAVs equipped with cameras oriented to the side (see Fig. 20 21 is realized repeatedly to show the robustness of the method. The formation flies twice to the target region and back to the initial position. The initial position from the first flight is considered the centre of the target region for the return flight, etc. The multi-criteria optimization problems defined in equations (11) and (12) were solved by sequential programming method (CFSQP toolbox Version 2.0) in the experiments. All MAVs are equipped with identification circle patterns for fast relative localization. The independent motion capture system (VICON) is used as a ground truth to evaluate the performance of the visual relative localization 
during the formation driving experiments. Complete records of the experiments are available in [39].

The main purpose of the experiment was to verify the ability of the system to relatively localize MAVs in a compact formation using VICON as a ground truth, and therefore, the experiment was realized in simple laboratory conditions. Nevertheless, the trajectory planning and formation stabilization mechanisms may be efficiently employed in more complex situations as was shown in our previous research with Unmanned Ground Vehicles (UGVs).

See our results [48], where performance of trajectory planning for UGV formations is shown in a complex office-like environment. In [49], complex maneuvers of the formation controlled by the MPC were presented. Usage of the MPCbased stabilization and trajectory planning in task of airport snow shoveling by fleets of autonomous ploughs is presented in [50] with stability analyses in [51]. The work in [52] is focused on testing the ability of the approach to avoid dynamic obstacles by integration of its motion prediction into the MPC trajectory planning.

The approach presented in this paper is an extension of the methods designed for UGVs taking into account constraints of the visual relative localization system, which is used for stabilization of MAV formations in 3D shapes. Due to the employed convex hull that represents the entire formation in the planning process, the trajectory planning ability of the system is not limited and it achieves a similar performance as was presented for UGVs. In case of limited computational power onboard of MAVs, where the complexity of the optimization is increased by the third dimension, the applicability of the method in real-time could be limited. In this case, the additional planning horizon needs to be decomposed as was proposed by the hierarchical approach in [53] for UGVs in convex environments.

\section{B. Scenario 2: Cooperative searching for extremes in a field of a measured physical value.}

The second scenario deals with searching for locations in a 3D environment with an extreme in a field of a measured physical value. In particular, the investigated scenario is motivated by searching for locations with a minimum GSM signal in mountain areas, which are hard to reach, but which need to be sufficiently covered for safety reasons. Another example can be monitoring the intensity of WiFi signal in industrial complexes, shopping malls or large office buildings. The signal coverage and interfaces from multiple transmitters can hardly be modelled in such complex 3D environments, and physical measurements are therefore unavoidable. With their fast deployment and operability, MAVs are especially appealing to provide the desired data in these large and complex areas. Moreover, swarm intelligence can speed up the process of searching for extremes in the measured intensity and can enable more autonomy within the system.

In the proposed system, we rely on a Fish Search School (FSS) technique [54], which allows us to define the swarm motion based on the actual state of particular particles. Each particle in the FSS defines its future movement based only

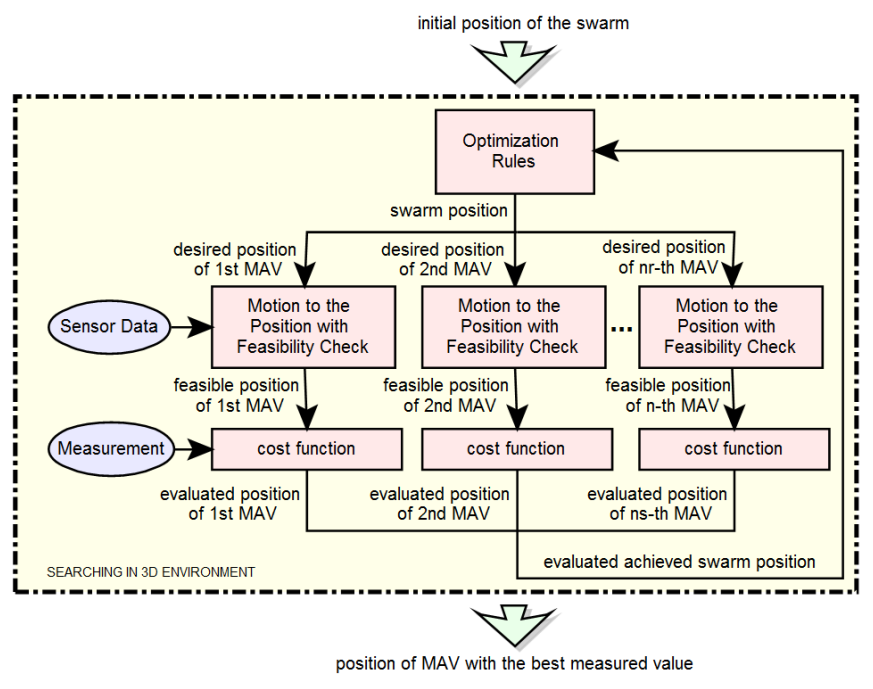

Fig. 22. Scheme of the system for feasible navigation of MAV swarms stabilized by visual relative localization based on the FSS algorithm.

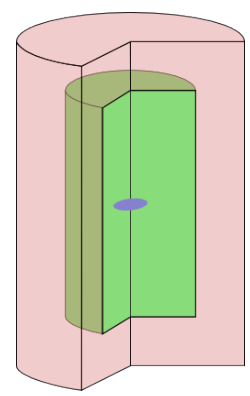

Fig. 23. Safety zones around a quadrocopter.

on its current state and the states of neighbors obtained by onboard systems. This is preferable to methods such as Particle Swarm Optimization (PSO), where the new desired positions of MAVs are determined based on the best achieved position of a particle of the swarm so far (the global best) and the best achieved positions of each particle (the personal best). This requires to remember or denote these locations in the environment. The FSS method can be directly used for control of a swarm of MAVs with the proposed relative localization considering each MAV as an FSS particle. In such a tangible FSS, MAVs may use odometry from IMU for the short term localization in the environment during the displacement between two consequent positions generated by the FSS rules. The required information on the position of neighbors is achieved by visual relative localization. Information about the global position of MAVs in the environment is not necessary, as the robots are, in a matter of fact, steered by the distribution of the measured signal intensity.

The FSS control rule is created by three simple operators:

1) individual movement,

2) collective-instinctive movement,

3) collective-volitive movement that depends on a factor describing the recent success of the swarm.

The success of the swarm is determined by the progress of 
the cost function values, which are provided by the sensory measurement in this application example (see a mathematical expression of these rules in [54]). In the proposed tangible FSS algorithm, the optimization vector represents the position of one MAV simply as $X=[x, y, z]$, in contrast to the PSO environment coverage presented in Section VI-C. where the positions of all $n_{r}$ MAVs are encoded into a unique optimization vector (the PSO particle). The number of FSS particles is equal to the number of physical robots $n_{r}$ in the swarm (in the PSO environment coverage algorithm the number of PSO particles is equal to the number of virtual MAV swarms).

A scheme of the tangible FSS algorithm is shown in Fig. 22. The core of the motion planning and group stabilization algorithm is in the Optimization Rules block, where the FSS control rules are implemented according to [54]. The input of this block is an estimate of the current relative positions of particles within the swarm and values of the cost function obtained for each MAV. In each optimization step, desired new positions of all MAVs are computed using the FSS rules based on this information. Then, the swarm is controlled into the new positions using the position control described in Section IV] while the localization and motion constraints are checked on the basis of the available sensor data (the Motion to the Position with Feasibility Check blocks).

The most important part of the Sensor Data block for swarm stabilization and inter-vehicle collision avoidance is an estimation of relative positions of neighbors provided by the onboard localization system. Based on this information, the actual shape of the swarm is considered feasible if none of the MAVs are within the safety zones of another MAV. This means that the shape is feasible regarding the intervehicle collisions and the air-flow effect from propellers of neighboring MAVs. Two safety zones are considered (see Fig. 23) for collision avoidance. Robots can temporarily (conditionally) enter the red outer zone, but once they reach the blue inner zone, which is considered forbidden, they have to return back to release from both zones before the next FSS iteration. The concept of two zones prevents the system from oscillations and deadlocks in applications with dense swarms, where close proximities of MAV pairs and even multiple MAVs occur frequently.

A similar concept is employed for keeping the robots within the range of the relative localization. Again, two limits on the maximal distance between relatively stabilized MAVs are considered. The weaker restriction can be temporarily broken. Both limits have to be satisfied before the next FSS step. This approach decreases the likelihood that the swarm evolution gets stuck if several MAVs move close to the borders of their safety zones or close to the limits of their relative localization. Once all MAVs approach the locations obtained by the FSS rules or reach the last feasible constellation, sensor measurements are taken in the new positions of swarm particles. The measured values act as the cost values of the FSS optimization. The cost function evaluation is represented by the cost function block in the figure. The obtained cost function values and the information on the relative positions of neighbours are used as input of the Optimization Rules block in the new FFS iteration.

As a stopping criteria a predefined maximum number of iterations is used in experiments presented in this article. According to [54], the progress of the total mass of FSS swarm and rate of the cost function values changes may be applied to detect termination of the searching process or deadlocks, but these studies go beyond the scope of this paper.

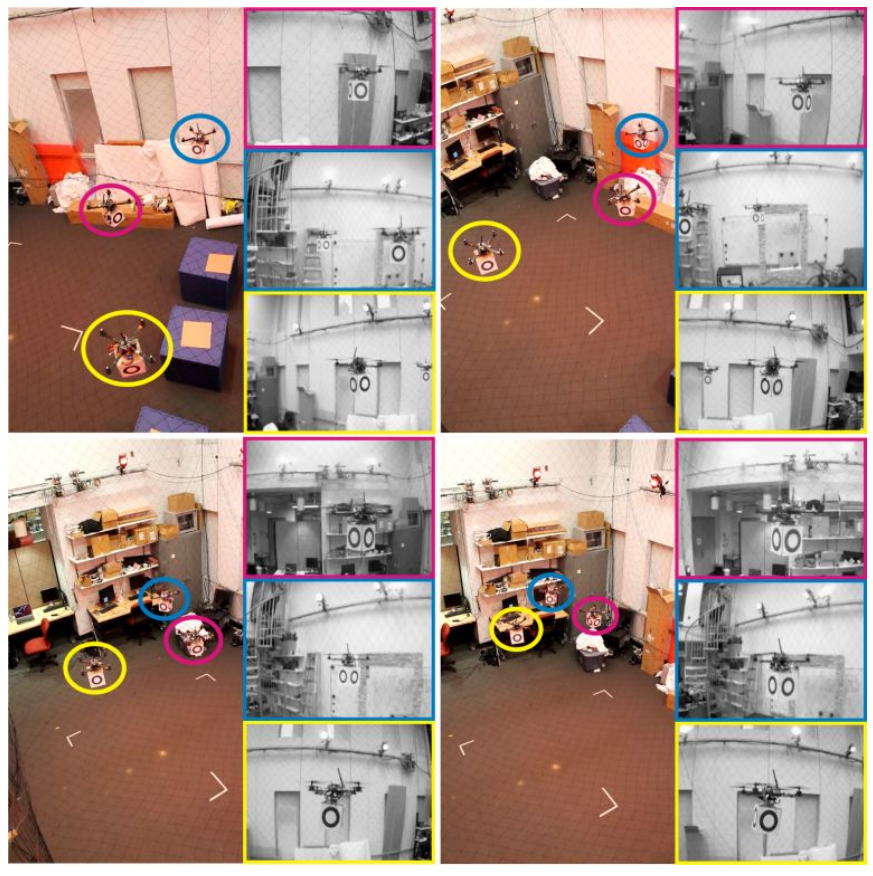

Fig. 24. Experiment with a swarm of 3 MAVs controlled by the feasible FSS rules.

1) Experimental evaluation of the planning technique in flight conditions.: The experiment in Fig. 24 25 demonstrates the use of the proposed tangible FSS method with onboard relative localization for searching in a 3D environment. In the experiments, MAVs cooperatively search for locations with the lowest intensity of a signal transmitted from four transmitters distributed in the environment at different altitudes. The intensity of the signal is simulated in the experiment based on known locations and the transmission power of the virtual transmitters. Instead of a real measurement of the signal strengths, the cost value for the $i$-th particle is then obtained as

$$
F(X)=\sum_{j=1}^{4}\left|x_{i}-s_{j}\right|^{-2},
$$

where $s_{j}$ is the location of the $j$-th transmitter.

The progress of minimal cost value 13 "measured" by an MAV of the group in the particular iteration is shown in Fig. 26. The temporary increase of the cost values at the beginning of the experiment is caused by the initial 


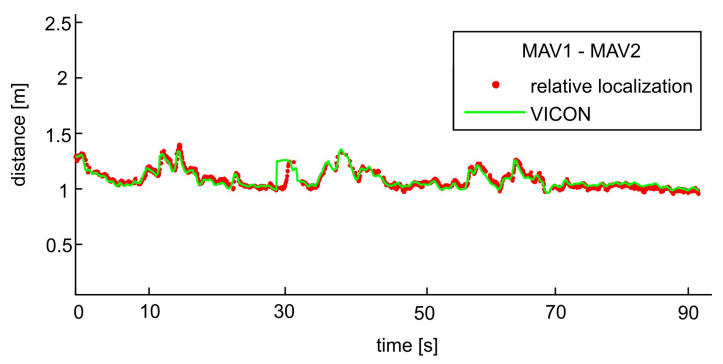

(a) Comparison of the relative distances between MAVs captured by the onboard vision system and by data obtained by VICON.

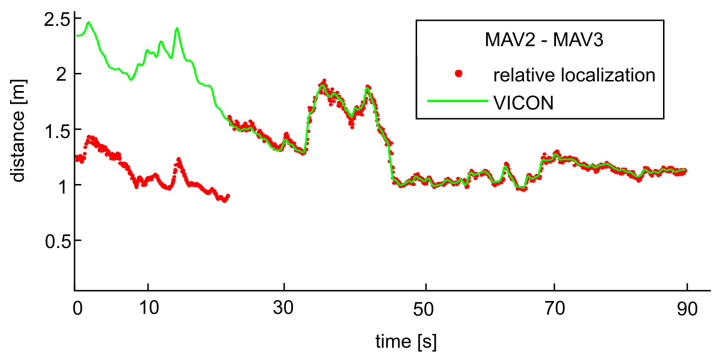

(b) Comparison of the relative distances between MAVs captured by the onboard vision system and by data obtained by VICON.

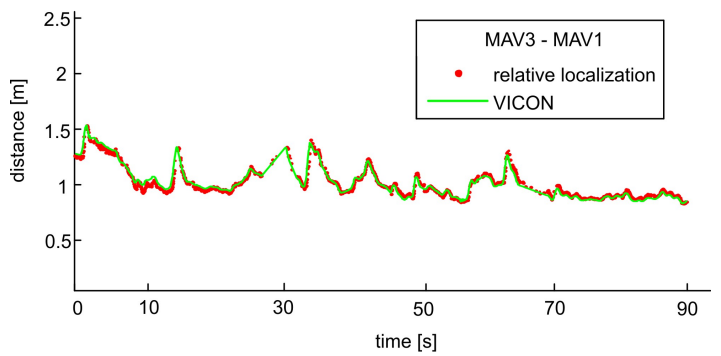

(c) Comparison of the relative distances between MAVs captured by the onboard vision system and by data obtained by VICON.
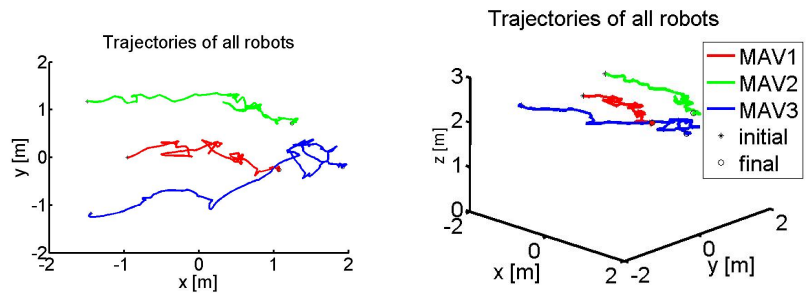

(d) Positions of MAVs captured by (e) 3D view of the positions of VICON during the experiment. MAVs captured by VICON during the experiment.

Fig. 25. Tangible Fish Search School (FSS) optimization. MAVs steered by FSS rules towards a location corresponding to a minimum of a signal transmitted from multiple transmitters. (slower movement of MAVs)

stabilization of the group into a shape that satisfies the constraints given by size of the MAVs and their relative localization. For evaluation of the FSS algorithm with tangible particles (the real MAVs), results of a simulation with dimensionless particles are also presented in Fig. 26. The

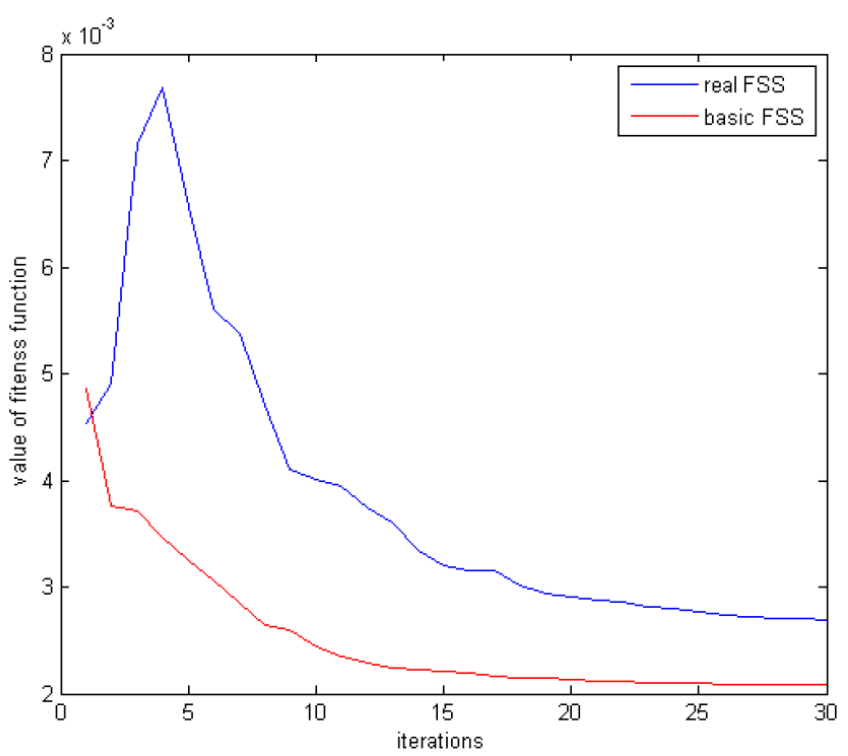

Fig. 26. Progress of values of the lowest intensity measured by a swarm member at the particular iteration (the cost function values) in the experiment with real FSS particles and in a simulation with basic FSS method using dimensionless particles.

simulation was run using the same map and initial setup as in the real experiment. In the simulation, the initial stabilization of the group is not necessary, since the basic FSS method without motion constraints is used and the cost function values decrease from the beginning of the searching process.

The results presented in Fig. 25 show that the requirements on the maximal relative distance between particular pairs of MAVs $(2.5 \mathrm{~m})$ are kept during the experiment, and that the neighboring MAVs are always in the view angle of the onboard cameras. The relative distance $\left|x_{i}-s_{j}\right|$ is obtained on the basis of data from the VICON motion capture system. In real-world deployment, knowledge of the global positions of MAVs, denoted as $x_{i}$ here, would not be necessary, since the tangible FSS technique requires only knowledge on the positions relative to neighbors (and obstacles) and the actual measured intensity, which can all be obtained by onboard sensors.

\section{Scenario 3: Environment coverage for cooperative surveillance.}

The third scenario demonstrates deployment of the proposed system in the task of cooperative surveillance (presence of MAVs at locations of interest). This section is a summary of the approach originally published by our team in the conference paper [55]. Here, the description of the method is put into the context of the presented control and localization system and it is used as an example of the system deployment in scenarios, where the trajectories of the robots have to be purposely computed prior the mission for their verification by the operator. In the scenario, a set of goals (areas) is assigned to a limited number of autonomous robots 
(MAVs) with the aim to find a static swarm configuration that can guard the areas. Let us call the set of static positions of all MAVs in the surveillance areas a swarm distribution, and let us call the complete task of the motion of MAVs from the initial depot into the static swarm distribution a swarm deployment.

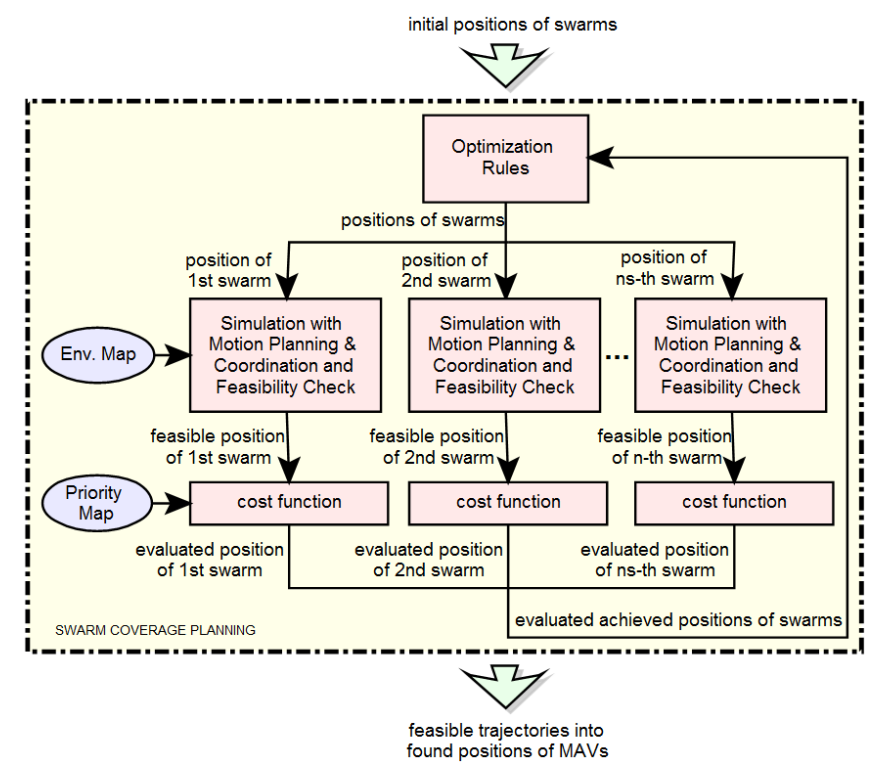

Fig. 27. Scheme of the planning system for environment coverage by MAV swarms stabilized by visual relative localization.

Again, the MAV swarm has to respect the motion, localization, and sensing constraints of MAVs. These constraints have to be applied in the final static swarm distribution and also during the swarm deployment. In the case that the swarm is not capable of covering the given set of locations of interest completely, for example because of an insufficient number of entities available or constraints on sensing, the coverage by the team members is maximised in the searching process. In this manner, we tackle the problem of static coverage of a set of areas by spreading a swarm of MAVs, while the swarm constraints are guaranteed for all obtained trajectories between the initial location of the MAVs into the achieved swarm distribution. So, we are looking for both: 1) the feasible static shape of the swarm (locations of particular swarm entities - the swarm distribution) and 2) a feasible plan of motion from the initial configuration to this target shape (trajectories for all MAVs - the swarm deployment). This leads us to a swarm-shape optimization with the need to keep the history (a feasible MAV movement) of swarm shape evolution from its initial state. This can be understood as a novel approach to multi-objective optimization, where a motion planning technique is integrated directly into the core of the optimization engine. The 3D pose of all MAVs in the swarm is then encoded into a unique optimization vector as $X=\left[x_{1}, y_{1}, z_{1}, x_{2}, y_{2}, z_{2}, \cdots, x_{n_{r}}, y_{n_{r}}, z_{n_{r}}\right]$, where $n_{r}$ is the number of robots in the swarm. The Particle Swarm Optimization (PSO) technique [56] is employed as the optimization method in this application.
A simple scheme of the proposed approach is shown in Fig. 27. In comparison with standard optimization techniques, where in each optimization step the actual solution (or several solutions) is directly evaluated by a cost function, here, the optimization vector is suited to respect the swarm constraints before the optimization continues. In each step of the optimization, the new shape of the swarm encoded into the optimization vector is used as an input to a motion planning approach, which generates collision-free trajectories connecting the desired positions with the actual state for each single MAV. The given plan is realized in a simulation using the trajectory tracking mechanism [57] with the MAV model introduced in Section IV. The simulation is run until the desired positions are reached or a violation of the swarm constraints is detected. If a mutual collision between MAVs is detected, the plan can often be corrected by a proper permutation of the goals assigned to particular vehicles. This does not influence the optimization process, since the MAVs are considered to be identical swarm particles. Any multi-robot coordination approach may be utilized in this phase of the planning mechanism if the permutation of goals is not sufficient. If a violation of the relative localization constraints (range, viewing angle, mutual MAV heading, etc.) is detected, the simulation is reversed into the last state considered as a feasible swarm distribution, and the optimization vector is replaced by this result. The achieved optimization vector is evaluated by the cost function, and the optimization continues in the next step from this state. An uncertainty in the optimization (e.g. the randomly weighted vectors addition in PSO) is crucial to increase the probability that the optimization will not end up in the same constraints violation, but it escapes from this potential deadlock.

In the experiments that are presented in this paper, the areas of interest are polygons and circles. The set of all these areas is represented by a square grid $A o I$ that covers the entire workspace with size of each cell $10 \mathrm{~cm}$ (the experimental workspace is shown in Fig. 30(f)p. The cells of $A o I$ that represent the areas of interest are initialized with the value 1 , while the zero cells represent regions not assigned as areas of interest. The no-fly zones and the borders of the operational area are denoted by the mission operator as a set of convex polygons. These polygons are dilated and represented by the Environment Map of the same size $(n, m)$ as the size of the $A o I$ matrix.

The cost function that evaluates particular solutions of the swarm spreading problem (position of all MAVs of the swarm) can be then expressed as

$$
f(X)=\sum_{x=1, y=1}^{m, n} \max \left(0, A o I_{x, y}-\sum_{i=1}^{n_{r}} R_{x, y, i} \frac{h_{o p t}^{2}}{h_{i}^{2}}\right)
$$

where $h_{i}$ is the height of the $i$-th MAV above the ground (the altitude) and $h_{\text {opt }}$ is the altitude determined as the "optimal" for the particular surveillance application. An MAV at lower altitude than $h_{\text {opt }}$ does not gain more information per square unit. The value of the variable $R_{x, y, i}$ is 1 if the cell of the 


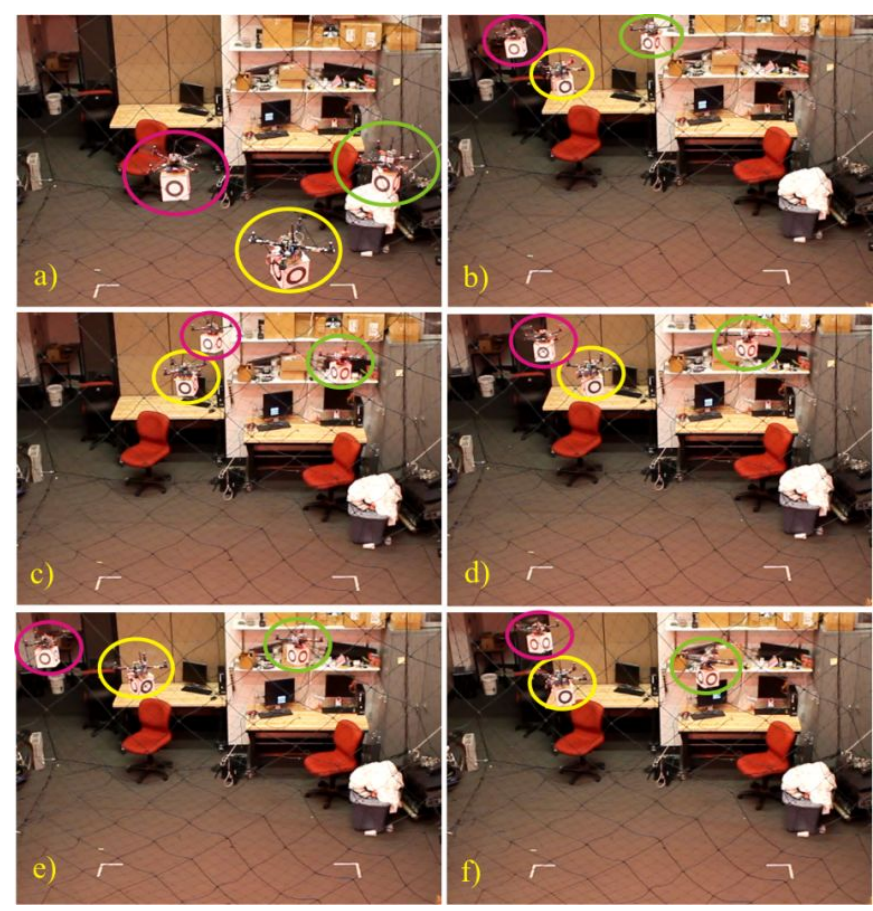

Fig. 28. Experiment with swarm of $3 \mathrm{MAVs}$ following trajectories obtained off-line by the proposed planning algorithm. MAVs are denoted by circles of different colours.

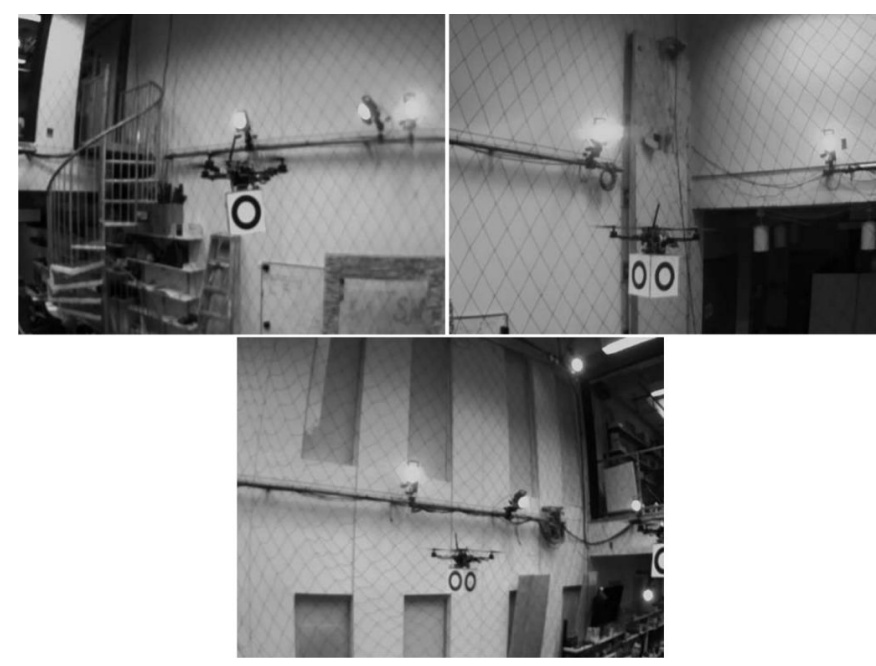

Fig. 29. Pictures taken by the onboard localization systems of all MAVs in the same moment (experiment in Fig. 28 .

workspace represented by the element $A o I_{x, y}$ is completely observed by the surveillance sensor of the $i$-th MAV in its position in the swarm and 0 in the opposite case.

Finally, we should emphasize that the proposed method does not guarantee to find the optimal distribution of the swarm and the optimal trajectories from the initial positions into the found locations. What is guaranteed is the feasibility of the solution with respect to the motion and localization constraints. Regarding the presented relative visual localization, it is important that the plan of the swarm distribution in the environment satisfies constraints given by the range of the relative localization and viewing angle of the onboard cameras, and that it respects the mutual heading of the MAVs.

1) Experimental evaluation of the planning technique in flight conditions.: The aim of the experiment in Fig. 28 30 is to demonstrate deployment of the proposed system in a surveillance task, where locations of interest with different priorities are covered by a self-stabilized swarm of MAVs. The feasibility of the plan for swarm distribution in the environment with known sets of areas of interest, no-fly zones and initial positions of the MAVs is verified in the experiment. The plan has to satisfy the constraints given by the range of the relative localization, the viewing angle of the on-board cameras, the mutual heading of the MAVs, and the movement constraints during deployment of the system. Fig. 30 shows that a guess of the relative position of neighboring vehicles is continuously provided during the flight, and that the limit on the relative distances within the swarm entities $(2.5 \mathrm{~m})$ is kept.

Finally, we should mention that a global localization system (such as GPS) is necessary to reach the surveillance locations of the group in applications of the approach designed for swarm deployment. In most of the scenarios with compact MAV swarms, such positioning system has lower precision in comparison with the relative distances between MAVs. Therefore, the more precise onboard relative localization needs to be employed to protect the swarm members from mutual collisions. Moreover in our approach, such a global localization technique may be used to localize only few robots of the group. In the experiment presented in this section, the global position is estimated from the visual odometry of one of the MAVs using the PX4Flow sensor, while the entire group is stabilized using the onboard relative localization system.

\section{Comparison of performance of the system in scenarios $1-3$}

The purpose of this section was to demonstrate possibility of deployment of multi-MAV teams in different robotic scenarios and to show advantages and disadvantages of the onboard relative localization system in different techniques of control of MAV-groups.

In the scenario 2, outputs of the visual relative localization system may be used directly in the FSS rules, and therefore, the performance of the system directly influences the planning process. The advantage of this approach is that it is very robust to inaccuracy of measurement of the relative distances, but the FSS method is sensitive to drop out of the system. Longer malfunctions of the onboard localization system cause interruption of the searching process and may even lead to inter-vehicle collisions.

On the contrary, the control system is robust to a drop out of the localization method and sensitive to inaccuracy in measurements of the relative positions in scenarios 1 and 3 . In these scenarios, the onboard visual relative localization approach is used to unify local reference frames of the MAVs. In case of a temporary drop out of the relative 


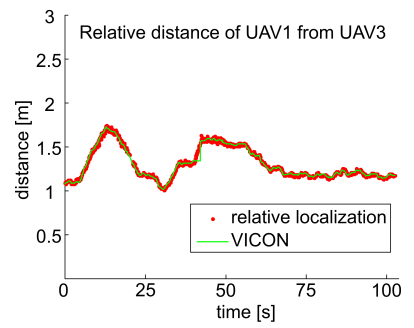

(a) Comparison of relative distances between MAVs captured by the onboard vision system and by the external motion capture system (VICON).

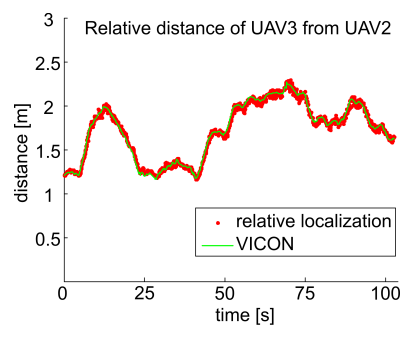

(c) Comparison of the relative distances between MAVs captured by the onboard vision system and by VICON.

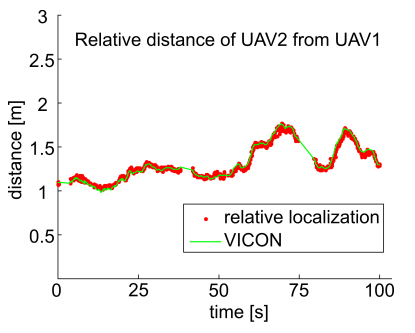

b) Comparison of the relative distances between MAVs captured by the onboard vision system and by VICON.

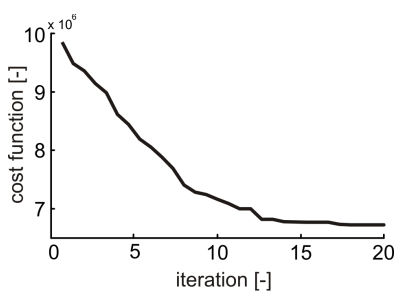

(d) Progress of the cost function values of the best PSO particle during off-line optimization of the swarm deployment found for the experiment in Fig. 28

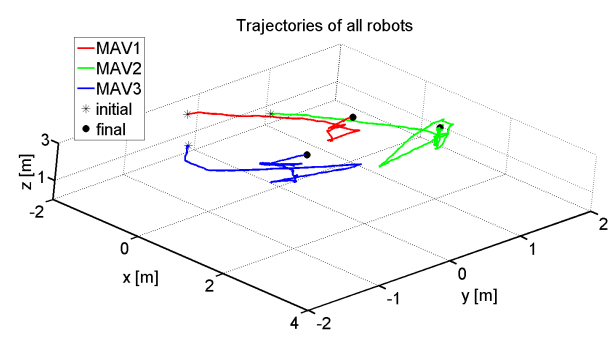

(e) 3D view of positions of MAVs captured by VICON during the experiment.

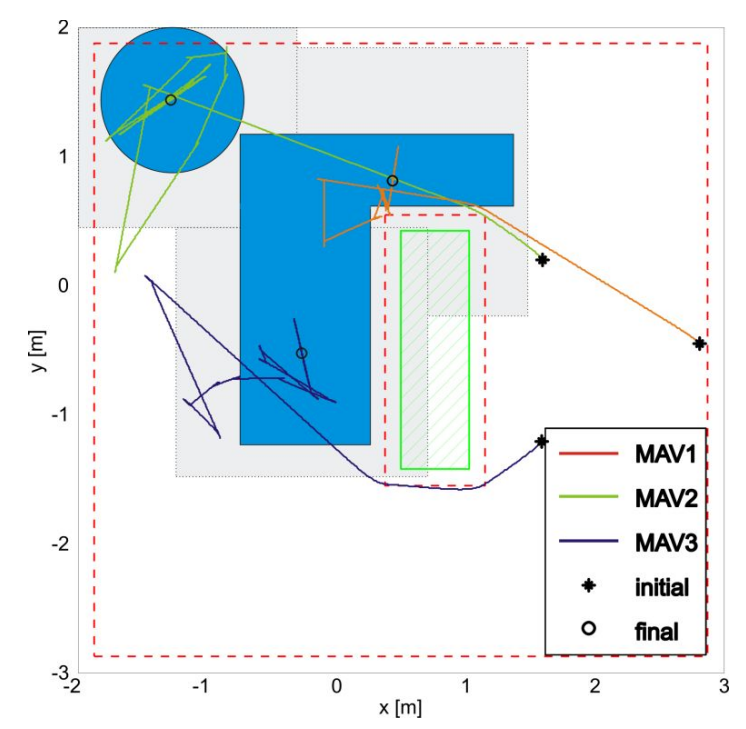

(f) Positions of MAVs captured by VICON during the experiment, with denoted areas of interest (blue regions) and the no-fly zone (green rectangle extended with a safety zone due to localization and control uncertainty).

Fig. 30. Swarm deployment in the environment to cover selected areas of interest. (experiment in Fig. 28,

localization, the MAVs can safely continue in their mission based on the visual odometry. The allowed duration of the drop out depends on the cumulative error of the odometry, the current distance between MAVs, the safety distance between MAVs, and the range of the onboard localization system. If the precision of the relative localization is low, the performance of formation flying and swarm deployment may be even worse than if the system relies only on the odometry of particular MAVs.

Finally, let us describe computational complexity and communication load required by these approaches.

The most computationally demanding is the scenario 1 , where the initial plan for the leader and also the control inputs for the followers need to be computed with sufficiently powerful PC to be able to get result between two planning steps. In the presented experiments, the initial plan was obtained in approximately $900 \mathrm{~ms}$ and each MPC step required 20-40ms. The plan was computed on an external PC (Intel Core i7, 8GB RAM) and then wirelessly sent into MAVs. This setup was sufficient for testing of the relative localization system, but MAVs already may be equipped by sufficiently small and powerful HW solutions to enable onboard computing. The data flow is low in this application, since only few control commands need to be sent into the MAVs in each control step. In case of onboard computing, which is expected in real applications, only the plan of the leader needs to be distributed within the team.

In the second scenario, the swarming algorithm is not computationally intensive and can be run onboard on the $\mu$-controller. Also the data flow is very low. Only the cost function value needs to be distributed within the team after each measurement, which is done with low update rate.

In the third scenario, the plan is purposely computed prior the mission to enable its verification by a human operator of the surveillance mission. Therefore, this plan may be obtained using standard PC and then sent into MAVs. The trajectory following process is run using the onboard ATmega $\mu$-controller as described in Section IV] During the flight, no communication is required except the initial synchronization command.

\section{CONCLUSION}

A complex system for stabilization and control of MAVgroups based on onboard visual relative localization has been presented in this paper. The aim of the system is to provide a tool for autonomous deployment of teams of unmanned quadrocopters in real world scenarios without the need for external localization. An onboard camera module with a fast image processing algorithm suited for the requirements of the group stabilization was described together with a simple controller using this module in the control feedback. As the core of the presented system, three various planning approaches have been proposed to solve specific multiMAV scenarios. The common factor of these methods is the endeavour to solve the group stabilization, motion planning and coordination tasks with the specific requirements given by the employed vision-based relative localization. 
The performance and feasibility of the motion planning methods presented here have been verified and evaluated by experiments with a fleet of MAVs. In the experiments, the performance of the onboard relative localization system in particular applications has been numerically evaluated with respect to an external motion capture system used as a ground truth.

In all motion planning approaches presented in this paper, the constraints on relative positions of the MAVs in the group are considered to satisfy the direct visibility among them and therefore to continuously keep the relative localization linkages during their motion. Nevertheless, various experiments of the system have shown that the relative localization of neighboring vehicles can be temporarily interrupted without any negative influence on the overall system stability. In our future work, we will integrate the possibility of temporal disconnection of the localization linkages, due to obstacles appearing in between of MAVs or due to a temporary enlargement of the group size, into the motion planning method. This significantly increases applicability of the system in GPS-denied environment, where the GPS signal is blocked by obstacles that may be present in such a high density that it is impossible to avoid them by the entire group, and where the temporary occurrence of the obstacles in between of the MAVs has to be allowed.

\section{ACKNOWLEDGMENTS}

This work has been supported by the Ministry of Education of the Czech Republic under project no. LH11053 and the experimental works required for paper revisions by project no. HS 13167/830/8301616C000 founded by Khalifa University for the MBZIRC competition, both projects supporting the joint research of the Czech Technical University in Prague and the University of Pennsylvania. In addition, Martin Saska has been supported by the Grant Agency of the Czech Republic under postdoc grant no. P103-12/P756. The work of Jan Faigl has been also partially supported by the Czech Science Foundations (GACR) under the research project no. 13-18316P. Tomas Krajnik has been supported by the EU project STRANDS (ICT-600623). Tomas Baca has been supported by CTU grant no. SGS15/157/OHK3/2T/13. Final experiments done by Martin Saska for revisions of the paper have been supported by the Czech Science Foundations (GACR) under the research project No. 16-24206S.

\section{REFERENCES}

[1] J. Faigl, T. Krajník, V. Vonásek, and L. Přeučil, "On Localization Uncertainty in an Autonomous Inspection," in IEEE Int. Conf. on Robotics and Automation (ICRA), 2012.

[2] W. Teacy, J. Nie, S. McClean, and G. Parr, "Maintaining connectivity in UAV swarm sensing," in IEEE GLOBECOM Workshops, 2010.

[3] T. Schmickl and K. Crailsheim, "Trophallaxis within a robotic swarm: bio-inspired communication among robots in a swarm," Autonomous Robots, vol. 25, pp. 171-188, 2008.

[4] A. Winfield, W. Liu, J. Nembrini, and A. Martinoli, "Modelling a wireless connected swarm of mobile robots," Swarm Intelligence, vol. 2, pp. 241-266, 2008.

[5] H. Hamann and H. Worn, "A framework of spacetime continuous models for algorithm design in swarm robotics," Swarm Intelligence, vol. 2, pp. 209-239, 2008.
[6] S. Berman, A. Halasz, M. Hsieh, and V. Kumar, "Optimized stochastic policies for task allocation in swarms of robots," IEEE Transactions on Robotics, vol. 25, no. 4, pp. 927 -937, 2009.

[7] W. Liu, A. Winfield, J. Sa, J. Chen, and L. Dou, "Strategies for energy optimisation in a swarm of foraging robots," in Swarm Robotics, 2007, vol. 4433, pp. 14-26.

[8] P. Fazli, A. Davoodi, and A. Mackworth, "Multi-robot repeated area coverage," Autonomous Robots, vol. 34, no. 4, pp. 251-276, 2013.

[9] R. K. Sharma and D. Ghose, "Collision avoidance between UAV clusters using swarm intelligence techniques," Intern. J. Syst. Sci., vol. 40, pp. 521-538, 2009.

[10] M. Kumar, D. Garg, and V. Kumar, "Segregation of heterogeneous units in a swarm of robotic agents," IEEE Transactions on Automatic Control, vol. 55, no. 3, pp. $743-748,2010$.

[11] H. Yu and R. Beard, "A vision-based collision avoidance technique for micro air vehicles using local-level frame mapping and path planning," Autonomous Robots, vol. 34, no. 1-2, pp. 93-109, 2013.

[12] M. Turpin, N. Michael, and V. Kumar, "Trajectory design and control for aggressive formation flight with quadrotors," Autonomous Robots, vol. 33, no. 1-2, pp. 143-156, 2012.

[13] M. Kloetzer and C. Belta, "Temporal logic planning and control of robotic swarms by hierarchical abstractions," IEEE Transactions on Robotics, vol. 23, no. 2, pp. $320-330,2007$.

[14] N. Cai, J.-X. Xi, and Y.-S. Zhong, "Swarm stability of high-order linear time-invariant swarm systems," Control Theory Applications, IET, vol. 5, no. 2, pp. 402-408, 2011.

[15] C. C. Cheah, S. P. Hou, and J. J. E. Slotine, "Region-based shape control for a swarm of robots," Automatica, vol. 45, no. 10, pp. 2406 $-2411,2009$.

[16] A. Marjovi and L. Marques, "Optimal spatial formation of swarm robotic gas sensors in odor plume finding," Autonomous Robots, vol. 35, no. 2-3, pp. 93-109, 2013.

[17] D. J. Bennet and C. R. McInnes, "Verifiable control of a swarm of unmanned aerial vehicles," Journal of Aerospace Engineering, vol. 223, no. 7, pp. 939-953, 2009.

[18] L. Barnes, R. Garcia, M. Fields, and K. Valavanis, "Swarm formation control utilizing ground and aerial unmanned systems," in IEEE/RSJ International Conference on Intelligent Robots and Systems, 2008.

[19] O. Holland, J. Woods, R. Nardi, and A. Clark, "Beyond swarm intelligence: the UltraSwarm," in IEEE Swarm Intelligence Symposium, 2005.

[20] L. Doitsidis, S. Weiss, A. Renzaglia, E. Kosmatopoulos, R. Siegwart, D. Scaramuzza, and M. Achtelik, "Optimal surveillance coverage for teams of micro aerial vehicles in GPS-denied environments using onboard vision," Autonomous Robots, vol. 33, no. 1-2, pp. 173-188, 2012.

[21] N. Leonard and E. Fiorelli, "Virtual leaders, artificial potentials and coordinated control of groups," in Proc. of the 40th IEEE Conference on Decision and Control, 2001.

[22] T. Balch and M. Hybinette, "Social potentials for scalable multi-robot formations," in Proc. of IEEE Conference on Robotics and Automation, 2000.

[23] V. Trianni, Evolutionary Swarm Robotics. Springer, 2008.

[24] A. Christensen, R. O'Grady, and M. Dorigo, "From fireflies to fault-tolerant swarms of robots," IEEE Transactions on Evolutionary Computation, vol. 13, no. 4, pp. 754 -766, 2009.

[25] A. Buerkle and S. Leuchter, "Development of micro UAV swarms," in Autonome Mobile Systeme 2009, ser. Informatik aktuell, 2009, pp. 217-224.

[26] D. H. Ballard, "Generalizing the Hough transform to detect arbitrary shapes," Pattern Recognition, vol. 13, no. 2, pp. 111-122, 1981.

[27] W. Cai, Q. Yu, and H. Wang, "A fast contour-based approach to circle and ellipse detection," in 5th World Congress on Intelligent Control and Automation (WCICA), 2004.

[28] A. A. Rad, K. Faez, and N. Qaragozlou, "Fast circle detection using gradient pair vectors," in DICTA, 2003.

[29] L.-Q. Jia, H.-M. Liu, Z.-H. Wang, and H. Chen, "An effective non-HT circle detection for centers and radii," in ICMLC, 2011.

[30] M. Carreras, P. Ridao, R. García, and T. Nicosevici, "Vision-based localization of an underwater robot in a structured environment," in ICRA, 2003.

[31] A. Masselli and A. Zell, "A Novel Marker Based Tracking Method for Position and Attitude Control of MAVs," in Proceedings of International Micro Air Vehicle Conference and Flight Competition, 2012. 
[32] L. Garca Carrillo, E. Rondon, A. Sanchez, A. Dzul, and R. Lozano, "Stabilization and trajectory tracking of a quad-rotor using vision," Journal of Intelligent \& Robotic Systems, vol. 61, pp. 103-118, 2011.

[33] M. Bošnak, D. Matko, and S. Blažič, "Quadrocopter control using an on-board video system with off-board processing," Robotics and Autonomous Systems, vol. 60, no. 4, pp. 657-667, Apr. 2012.

[34] S. Lange, N. Sunderhauf, and P. Protzel, "A vision based onboard approach for landing and position control of an autonomous multirotor uav in GPS-denied environments," in International Conference on Advanced Robotics (ICAR), 2009.

[35] S. Yang, S. A. Scherer, and A. Zell, "An onboard monocular vision system for autonomous takeoff, hovering and landing of a micro aerial vehicle," in International Conference on Unmanned Aircraft Systems (ICUAS'12), 2012.

[36] T. Krajník, M. Nitsche, J. Faigl, P. Vanek, M. Saska, L. Přeučil, T. Duckett, and M. Mejail, "A practical multirobot localization system," Journal of Intelligent \& Robotic Systems, p. Online first., 2014. [Online]. Available: http://dx.doi.org/10.1007/s10846-014-0041-x

[37] S. Yang, S. Scherer, and A. Zell, "An Onboard Monocular Vision System for Autonomous Takeoff, Hovering and Landing of a Micro Aerial Vehicle," Journal of Intelligent \& Robotic Systems, vol. 69, no. 1-4, pp. 499-515, Sep. 2012.

[38] J. Faigl, T. Krajník, , J. Chudoba, L. Preucil, and M. Saska, "Low-cost embedded system for relative localization in robotic swarms," in Proc. of IEEE International Conference on Robotics and Automation, 2013.

[39] Multimedia, "Various experiments with multi-MAV system verifying the proposed approach. http://mrs.felk.cvut.cz/data/mavgroups/ [cit. 2015-12-8]." 2015.

[40] N. Michael, D. Mellinger, Q. Lindsey, and V. Kumar, "The grasp multiple micro-UAV testbed," IEEE Robotics Automation Magazine vol. 17, no. 3, pp. 56-65, Sept 2010.

[41] T. D. Barfoot and C. M. Clark, "Motion planning for formations of mobile robots," Robotics and Autonomous Systems, vol. 46, pp. 65-78, 2004.

[42] M. Saska, V. Vonasek, T. Krajnik, and L. Preucil, "Coordination and Navigation of Heterogeneous MAV\&UGV Formations Localized by a hawk-eye-like Approach Under a Model Predictive Control Scheme," International Journal of Robotics Research, vol. 33, no. 10, pp. 13931412, 2014.

[43] M. Saska, T. Krajnik, V. Vonasek, Z. Kasl, V. Spurny, and L. Preucil, "Fault-Tolerant Formation Driving Mechanism Designed for Heterogeneous MAVs-UGVs Groups,' Journal of Intelligent and Robotic Systems, vol. 73, no. 1-4, pp. 603-622, January 2014.

[44] M. Saska, Z. Kasl, and L. Preucil, "Motion Planning and Control of Formations of Micro Aerial Vehicles," in Proceedings of The 19th World Congress of the International Federation of Automatic Control, 2014.

[45] Z. Chao, S.-L. Zhou, L. Ming, and W.-G. Zhang, "UAV formation flight based on nonlinear model predictive control," Mathematical Problems in Engineering, vol. 2012, no. 1, pp. 1-16, 2012.

[46] T. S. No, Y. Kim, M.-J. Tahk, and G.-E. Jeon, "Cascade-type guidance law design for multiple-UAV formation keeping," Aerospace Science and Technology, vol. 15, no. 6, pp. 431 - 439, 2011.

[47] C. Liu, W.-H. Chen, and J. Andrews, "Piecewise constant model predictive control for autonomous helicopters," Robotics and Autonomous Systems, vol. 59, no. 78, pp. 571 - 579, 2011.

[48] M. Saska, J. S. Mejia, D. M. Stipanovic, and K. Schilling, "Control and navigation of formations of car-like robots on a receding horizon," in Proc of 3rd IEEE Multi-conference on Systems and Control, 2009.

[49] M. Saska, J. Mejia, D. Stipanovic, V. Vonasek, K. Schilling, and L. Preucil, "Control and Navigation in Manoeuvres of Formations of Unmanned Mobile Vehicles," European Journal of Control, vol. 19, no. 2, pp. 157-171, 2013.

[50] M. Saska, V. Vonasek, and L. Preucil, "Control of ad-hoc formations for autonomous airport snow shoveling," in IEEE/RSJ International Conference on Intelligent Robots and Systems, 2010, vol. 1. Taipei: IEEE Industrial Electronics Society, 2010, pp. 4995-5000.

[51] _ _ "Trajectory Planning and Control for Airport Snow Sweeping by Autonomous Formations of Ploughs," Journal of Intelligent and Robotic Systems, vol. 72, no. 2, pp. 239-261, 2013.

[52] M. Saska, V. Spurny, and V. Vonasek, "Predictive control and sta- bilization of nonholonomic formations with integrated spline-path planning," Robotics and Autonomous Systems, vol. 75, 2016.

[53] M. Saska, M. Hess, and K. Schilling, "Hierarchical spline path planning method for complex environments," in Proc. of the 4th International Conference on Informatics in Control, Automation and Robotics., Angers, France, 2007.

[54] C. Filho, F. Lima Neto, A. Lins, A. Nascimento, and M. Lima, "Fish school search," in Nature-Inspired Algorithms for Optimisation, Studies in Computational Intelligence, vol. 193, 2009, pp. 261-277.

[55] M. Saska, J. Chudoba, L. Preucil, J. Thomas, G. Loianno, A. Tresnak, V. Vonasek, and V. Kumar, "Autonomous Deployment of Swarms of Micro-Aerial Vehicles in Cooperative Surveillance," in Proceedings of 2014 International Conference on Unmanned Aircraft Systems (ICUAS), 2014

[56] J. Kennedy and R. Eberhart, "Particle swarm optimization," in Proceedings of the IEEE International Conference on Neural Networks, vol. 4, 1995.

[57] T. Lee, M. Leoky, and N. McClamroch, "Geometric tracking control of a quadrotor UAV on se(3)," in 49th IEEE Conference on Decision and Control $(C D C), 2010$. 